GA-A16140

\title{
DOPPLER ION PROGRAM DESCRIPTION
}

\author{
by
P. HENLINE
}

MASTER

\section{DECEMBER 1980}

\section{GENERAL ATOMIC COMPANY}




\section{DISCLAIMER}

This report was prepared as an account of work sponsored by an agency of the United States Government. Neither the United States Government nor any agency Thereof, nor any of their employees, makes any warranty, express or implied, or assumes any legal liability or responsibility for the accuracy, completeness, or usefulness of any information, apparatus, product, or process disclosed, or represents that its use would not infringe privately owned rights. Reference herein to any specific commercial product, process, or service by trade name, trademark, manufacturer, or otherwise does not necessarily constitute or imply its endorsement, recommendation, or favoring by the United States Government or any agency thereof. The views and opinions of authors expressed herein do not necessarily state or reflect those of the United States Government or any agency thereof. 


\section{DISCLAIMER}

Portions of this document may be illegible in electronic image products. Images are produced from the best available original document. 


\section{DISCLAIMER}

This report was prepared as an account of work sponsored by an agency of the United States Government. Neither the United States Government nor any agency thereof, nor any of their employees, makes any warranty, express or implied, or assumes any legal liability or responsibility for the accuracy, completeness, or usefulness of any information, apparatus, product, or process disclosed, or represents that its use would not infringe privately owned rights. Reference herein to any specific commercial product, process, or service by trade name, trademark, manufacturer, or otherwise, does not necessarily constitute or imply its endorsement, recommendation, or favoring by the United States Government or any agency thereof. The views and opinions of authors expressed herein do not necessarily state or reflect those of the United States Government or any agency thereof. 
GA-A16140

\title{
DOPPLER ION PROGRAM DESCRIPTION
}

\author{
by \\ P. HENLINE
}

\author{
Work Supported by \\ Department of Energy \\ Contract DE-AT03-76ET51011
}

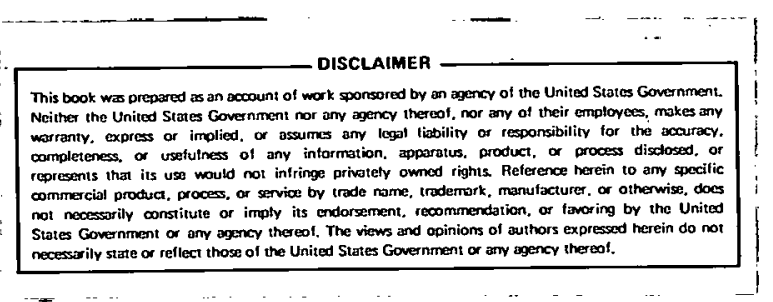

\section{GENERAL ATOMIC PROJECT 3344.110.720 DECEMBER 1980}

\section{GENERAL ATOMIC COMPANY}




\section{CONTENTS}

1. INTRODUCTION . . . . . . . . . . . . . . . . . . . 1

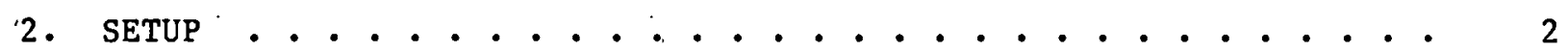

2.1. General ...................... 3

2.2. User Information . . . . . . . . . . . . . . . 3

3. ACQUIR . . . . . . . . . . . . . . . . . . . . . 11

3.1. General .. . . . . . . . . . . . . . . . . 11

3.2. Detailed Acquisition Program Description . . . . . . . . 12

4. PROGRAM PLTDAT . . . . . . . . . . . . . . . . . 23

4.1. General .. . . . . . . . . . . . . . . 23

4.2. Program Operation ................ . . 23

APPENDIX A . . . . . . . . . . . . . . .... 39

APPENDIX B . . . . . . . . . . . . . . . . . . 4 41

APPENDIX C . . . . . . . . . . . . . . . . .... . 4 46

APPENDIX D . . . . . . . . . . . . . . . . . . . . 4 48

APPENDIX E . . . . . . . . . . . . . . . . . . 54 


\section{FIGURES}

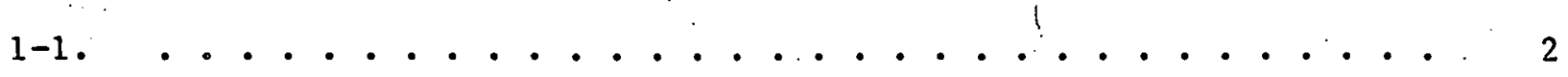

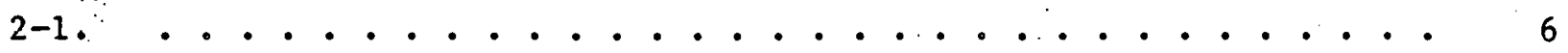

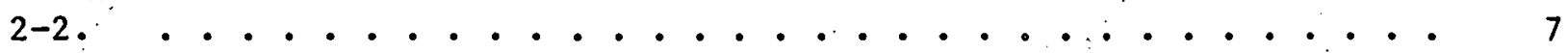

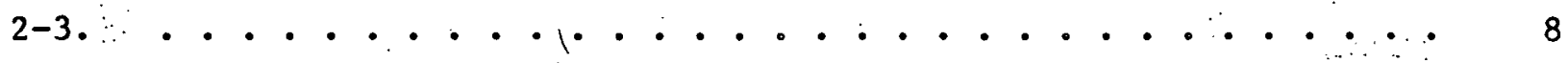

2-4. . . . . . . . . . . . . . . . . . . . 9

2-5. . . . . . . . . . . . . . . . . . . ... 10

3-1. . . . . . . . . . . . . . . . . . 13

4-1. . . . . . . . . . . . . . . . . . . . . 25

4-2. . . . . . . . . . . . . . . . . . . 26

4-3. . . . . . . . . . . . ........... . 27

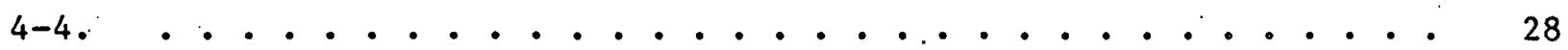

4-5. . . . . . . . . . . . . . . . . . . . . 31

4-6. . . . . . . . . . . . . . . . . . . 33

4-7. . . . . . . . . . . . . . . . . . . 34

4-8. . . . . . . . . . . . . . . . . . . 36

4-9. ............................ 37 
ABSTRACT

The Doppler spectrometer is a conventional Czerny-Turner grating spectrometer with a 1024 channel multiple detector. Light is dispersed across the detector, and its output yields a spectrum covering approximately $200 \AA$. The width of the spectral peak is directly proportional to the temperature of the emitting ions, and determination of the impurity ion temperature allows one to infer the plasma ion temperature. . The Doppler ion software system developed at General Atomic uses a TRACOR Northern $1710-31$ and an LSI-11/2. The exact configuration on Doublet III is different from TRACOR Northern systems at other facilities.

A subset of the TRACOR commands is used for control of data acquisition, storage and analysis. The system is powerful and flexible because most of its important functions are controlled by the LSI-11. The system acquisition parameters are easily updated and programs are written in a high-level language. Data analysis can be done separately from data acquisition, or some analysis can be done between shots. Normally, the acquisition program runs unattended (except for occasional replacement of a floppy disk), with analysis done later. The analysis program runs on any LSI-11/2 computer system. The analysis program produces various plots of the data. Most calculations are automatic, but the user must indicate the straight line fit to be used for the final ion temperature calculation.

other data refinements include smoothing, subtracting a background spectrum from a given spectrum, and subtracting a straight line from a given spectrum to minimize peak overlap. 


\section{INTRODUCTION}

The Doppler Ion experiment uses 3 programs which run on an LSI-11/2 16-bit minicomputer.

Program SETUP maintains a disk file which contains information about the experiment's parameters. This includes trigger times, exposure time, spectrometer wavelength, comments, gain setting, and tables needed for calculation.

Program ACQUIR uses some of the information in the setup file to initialize the Tracor-Northern 1710, prepare for shot firing, read the shot data from the $\mathrm{TN}-1710$, and write it to disk.

Program PLTDAT analyses any chosen shot. The program can do any of the following operations:

1. Plot all of the spectra received for a shot.

2. Identify peaks at user selected locations of one spectrum.

3. Plot a selected channel vs time and optionally do a range search on the selected channel.

4. Chose a region of one spectrum to expand and optionally do background subtraction, smoothing, and fitting.

5. The fit (if chosen) lets the operator manually fit the plot of in (I) vs $(\Delta \lambda)^{2}$ with a straight line. The data may be non-gaussian. Ion temperature is computed and calibration of full width at half $\max$ is done.

Figure 1-1 shows the hardware configuration. 


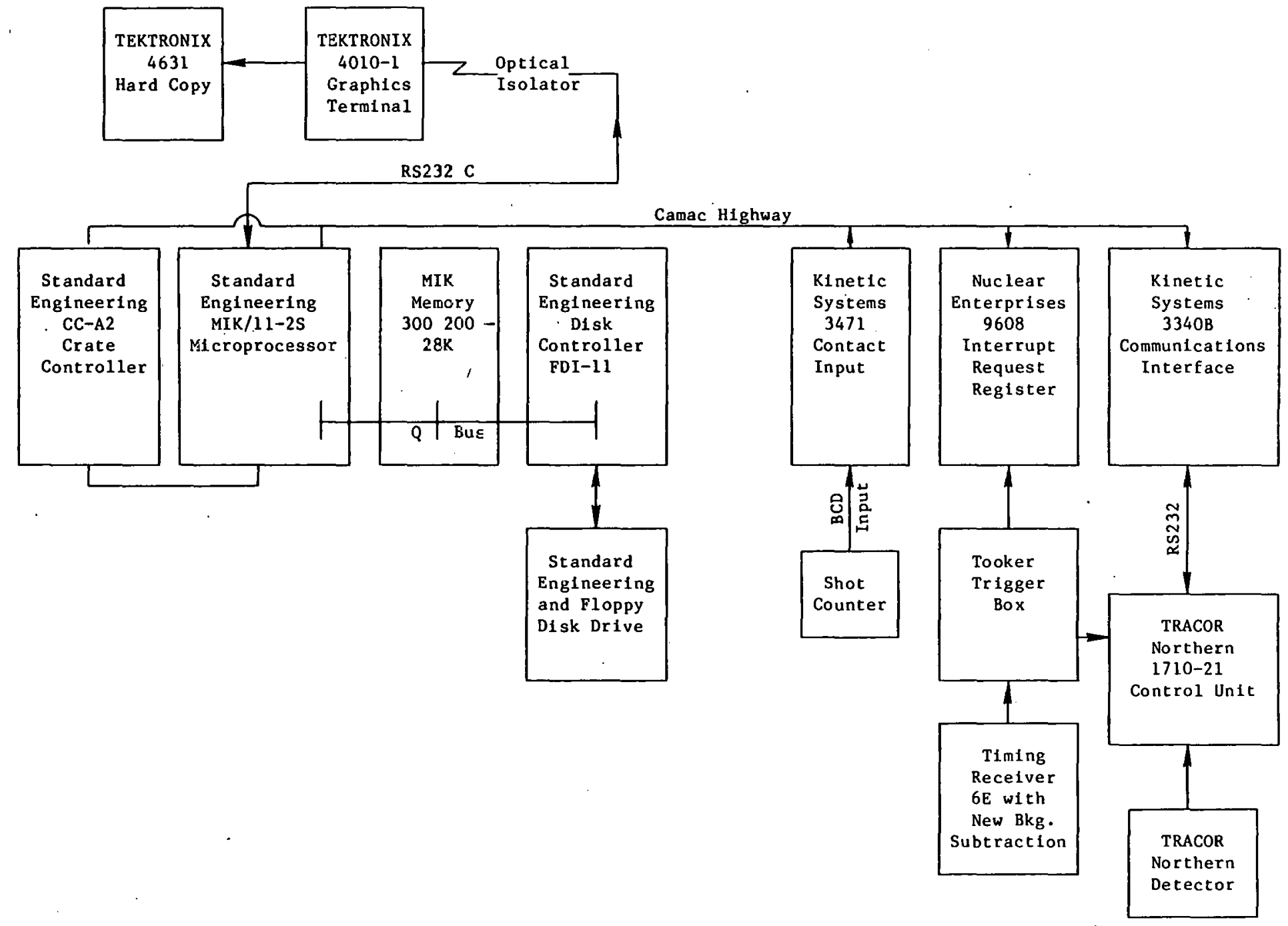




\section{SETUP}

\subsection{GENERAL}

This program maintains a file of data which describe parameters for the experiment. The program SETUP can update either DXO:SETUP.DAT or DXl:shotno.SET.

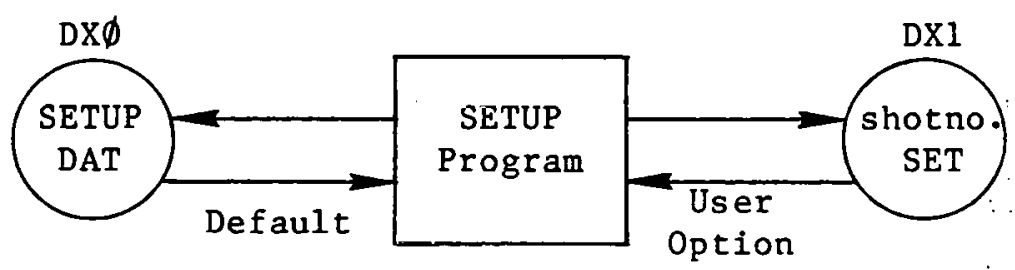

The file SETUP.DAT is the general purpose file which has experiment parameters. It is read before each shot to configure the Tracor Northern. Each parameter in the file can be changed individually, allowing the file to be updated between shots.

The file shotno.SET is the file which contains the setup data used for a particular shot. This information is needed to analyse each shot and must be kept with the shot data. However, there may be some occasions when the dispersion or full width half max tables need to be updated. The setup program can also update this file when the desired shot number is entered.

\subsection{USER INFORMATION}

To run the program on the terminal, type $R$ SETUP. The default mode is to update and/or list the file SETUP.DAT. Most updating will normally be done to this file. Typing EXIT will write the changes (if any) to tisk and end running the program. 
Figure 2-1 shows all of the Update commands which can be used. Most of the parameter usage is self-evident once the command is entered. The 'greater than' sign ( $>$ ) is the prompt character. 'Figure 2-2 shows the. screen after typing VIEW. All parameters are listed on one page. VIEW may be typed any time during updating and will display ail the changes currently entered. The changes are not written to disk until a WRIT or EXIT command is entered.

Figures 2-3 through 2-5 show sample usage of most commands. Lines typed by the user have a line to the left margin.

To update a file shotno.SET, commands must be entered in the following sequence:

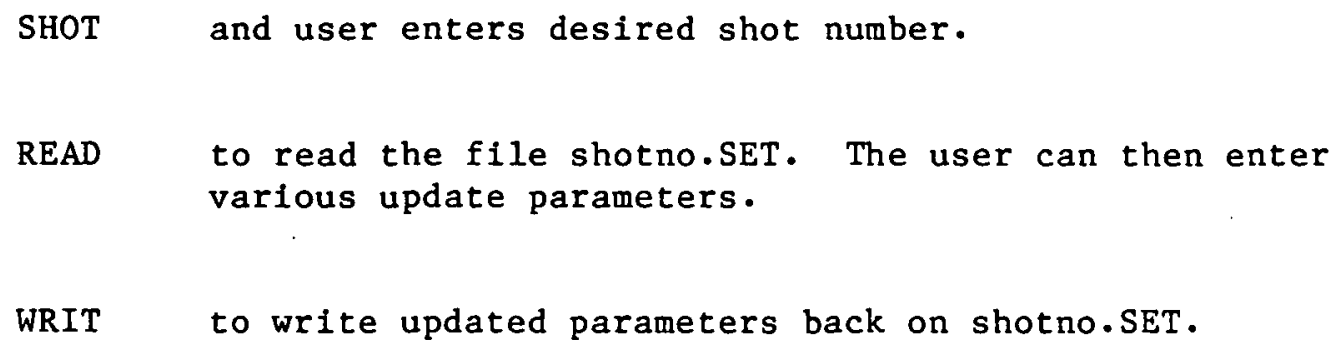

File Description for SETUP.DAT and Shotno.SET

The entire file is written as one binary record.

\begin{tabular}{|c|c|c|}
\hline $\begin{array}{l}\text { MONTH, DAY, YEAR } \\
\text { HOUR, MINUTE, SECOND }\end{array}$ & $\begin{array}{c}\text { Integer } \\
\qquad\end{array}$ & $\begin{array}{c}\text { Setup file date created or updated } \\
" \quad " \text { time } " ~\end{array}$ \\
\hline GAIN & Real & Gain setting (from TN helipot knob) \\
\hline $\mathrm{AM}^{*}$ & Integer & Acquisition mode \\
\hline $\mathrm{AS}^{*}$ & $"$ & Array size code \\
\hline AsX & $"$ & Actual array size implied by code \\
\hline $\mathrm{R}^{*}$ & " & Resolution code \\
\hline $\mathrm{RX}$ & $\because$ & Actual value of resolution \\
\hline
\end{tabular}

${ }^{*}$ See Tracor Northern manual, Appendix C, this report. 


\begin{tabular}{|c|c|c|}
\hline Preset value $1^{*}$ & Integer & $\mathrm{AM}+\mathrm{AS}+\mathrm{R}$ \\
\hline Preset value $2^{*}$ & " & Assumed to be 1 \\
\hline Preset value $3^{*}$ & $"$ & Integral - assumed to be 0 \\
\hline Exposure time & Real & Exposure time (in seconds) \\
\hline Preset value $4^{*}$ & Integer & $\begin{array}{l}\text { Calculated from exposure time. This } \\
\text { value is not unique but the TRACOR } \\
\text { will still respond correctly }\end{array}$ \\
\hline Preset value $5^{*}$ & $"$ & 0 \\
\hline Preset value $6^{*}$ & $"$ & 1 \\
\hline ITM & $"$ & $\begin{array}{l}\text { Number of triggers received from } \\
\text { Tooker trigger box }\end{array}$ \\
\hline $\operatorname{ITG}(16)$ & & $\begin{array}{l}\text { Times at which Tooker triggers occur } \\
\text { (milliseconds) }\end{array}$ \\
\hline IWAVE & $"$ & Spectrometer wave length setting, $\AA$ \\
\hline $\operatorname{IWVCM}(30)$ & " & $\begin{array}{l}60 \text { character comment related to } \\
\text { wavelength }\end{array}$ \\
\hline SLIT & Real & $\begin{array}{l}\text { Slit size of spectrometer entrance } \\
\text { slit, microns }\end{array}$ \\
\hline $\operatorname{IFILT}(30)$ & Integer & $\begin{array}{l}60 \text { character comment related to } \\
\text { wave length filter }\end{array}$ \\
\hline $\operatorname{ICOM}(30)$ & $"$ & 60 character general comment \\
\hline NDISP & 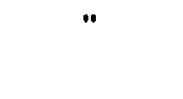 & $\begin{array}{l}\text { Number of entries in dispersion } \\
\text { table }\end{array}$ \\
\hline DISP(20) & Real & $\begin{array}{l}\text { First order dispersion values, in } \\
\text { A/channel. It is assumed that the } \\
\text { first value corresponds to lambda }= \\
1000 \text {, next for } 2000 \text {, etc. }\end{array}$ \\
\hline NTSEC & Integer & $\begin{array}{l}\text { Number of entries in full width half } \\
\text { max table. These are instrumental } \\
\text { corrections. }\end{array}$ \\
\hline $\operatorname{ITSEC}(10)$ & $"$ & $\begin{array}{l}\text { Times (in msec) for each of the FWHM } \\
\text { entries. }\end{array}$ \\
\hline FWHM(10) & Real & FWHM values in channels \\
\hline
\end{tabular}


TRACOR NORTHERN SETUP COMMANDS

\author{
HELP LIST THIS HELP \\ VIEW VIEW CURRENT DATA FILE INCLUDING MODIFICATIONS \\ EXIT EXIT - AUTOMATICALLY WRITE FILE IF CHANGES MADE \\ QUIT EXIT WITHOUTWRITING FILE \\ WRIT WRITE DATA FILE TO DISK \\ READ READ DATA FILE FOR THIS SHOT NUMBER OR MASTER \\ PARAMETERS \\ FWHM PROMPT FOR EDITING FWHM TABLE \\ DISP PROMPT FOR EDITING OF DISPERSION TABLE \\ SHOT ENTER SHOT NUMBER FOR A PARTICULAR FILE, \\ DEFAULT IS NON-SHOT NUMBER MASTER FILE \\ GAIN GAIN \\ ET EXPOSURE TIME \\ TRIG TRIGGER TIMES WITH PROMPTS \\ RSLN RESOLUTION CODE-HI OR LO \\ SLIT SLIT SIZE \\ ID LINE IDENTIFICATION WITH PROMPTS \\ FILT FILTER COMMENTS \\ $>$ \\ COMM GENERAL COMMENTS
}

Fig. 2-1. Tracor Northern setup commands 


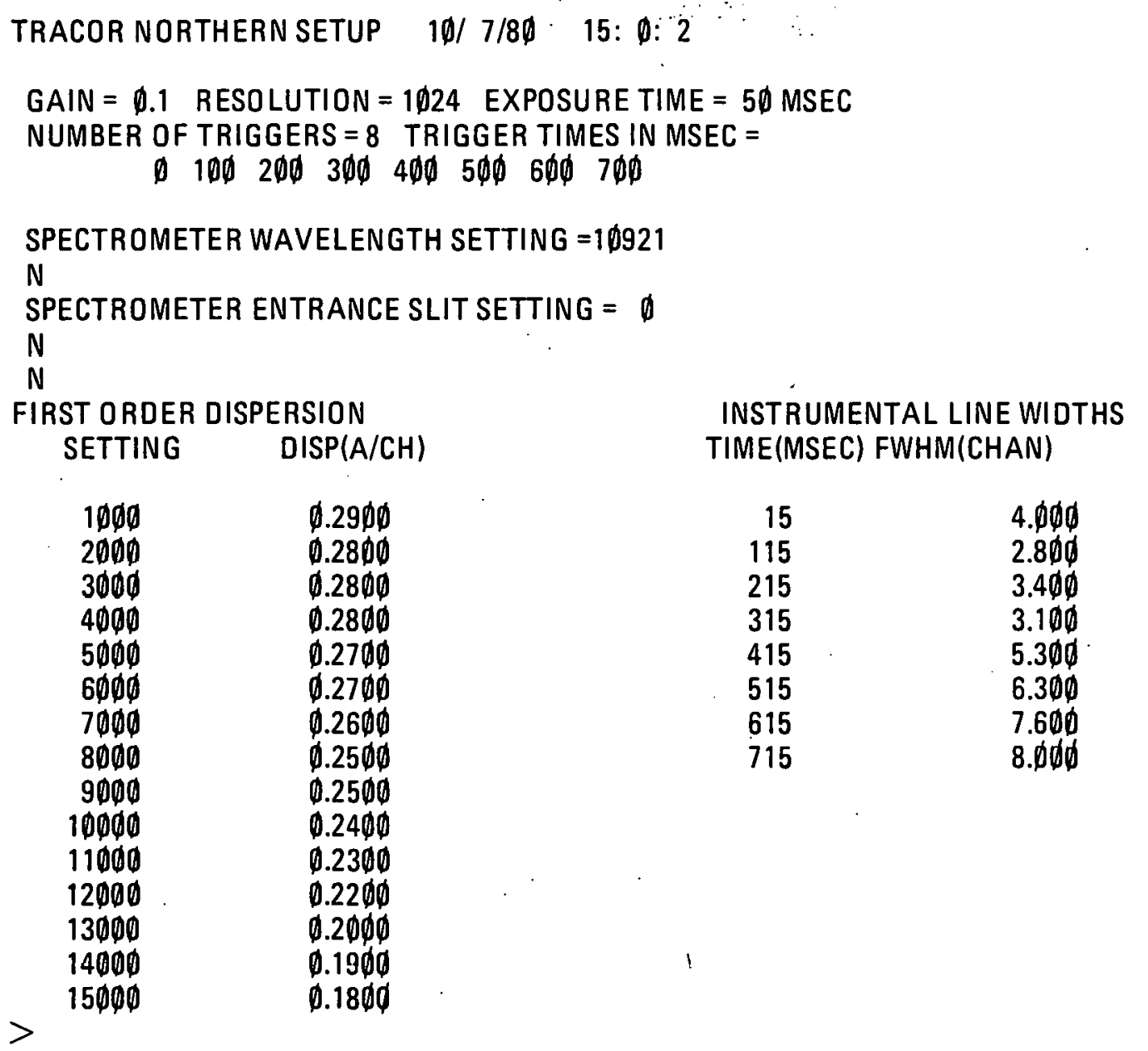

Fig. 2-2. Tracor Northern setup file contents 
- FWHM

DO YOU WANT.TO ENTER ENTIRE FWHM TABLE-Y OR N $Y$

ENTER NUMBER OF TIME SLOTS - MAX $=10$

$-3$

ENTER TIME (MSEC) AND FWHM V.ALUE FOR SLOT \# 1

$-153.8$

ENTER TIME (MSEC) AND FWHM VALUE FOR SLOT \# 2

$-1154.1$

ENTER TIME (MSEC) AND FWHM VALUE FOR SLOT \# 3

$-2154.2$

$>$

QUIT

- DISP

DO YOU WANT TO ENTER ENTIRE DISPERSION TABLE-Y OR N $-N$

GIVE ENTRY NUMBER YOU WISH TO CHANGE(MAX $=2 \phi)$ OR $\emptyset$

$-3$

ENTER DISPERSION VALUE FOR REGISTER SETTING OF $3 \emptyset \emptyset \emptyset$

$-.28$

GIVE ENTRY NUMBER YOU WISH TO CHANGE(MAX $=2 \varnothing)$ OR $\emptyset$

$-\stackrel{0}{>}$

Fig. 2-3. User interaction with setup program 
GAIN

OLD GAIN $=\emptyset .10$ ENTER GAIN OR RETURN

(RETURN)

$>$

OLD ET $=5 \emptyset$ MSEC ENTER ET OR RETURN (RETURN)

$>$

OLD SPEC SLIT ENTRANCE SETTING = ENTER SLIT SETTING OR RETURN (RETURN)

$>$

Fig. 2-4. User interaction with setup program 
- ID

CURRENT IDENTIFICATION VALUES = $10921 \mathrm{~N}$

INPUT CODE FOR LINE LABEL \& REGISTER SETTING

7 OTHER

1 NI XII 2818A FOURTH 11272

20 VII 1623A SIXTH 9740

3 C V 2271A FIFTH 11355

$40 \quad V \quad$ 2781A FOURTH 11124

5 C IV 1548A EIGHTH 12386

60 II 4415 A THIRD 13245

$-5$

$>$

TRIG

CHANGE TRIGGER TIMES - Y OR N ???

CURRENT NO. OF TRIGGERS $=8$ TIMES ARE IN MSEC $\emptyset \quad 100200300 \quad 400500600700$

$-Y$

ACCEPT DEFAULT TRIGGERS-Y OR N ??? DEFAULT IS 0 TRIGGERS AT $0,100, . ., 700$ MSEC

$-N$ ENTER NUMBER OF TRIGGERS

$-2$ ENTER TIME FOR TRIGGER NO: 1 IN MSEC

$-120$

ENTER TIME FOR TRIGGER NO. 2 IN MSEC

$-220$

$>$

Fig. 2-5. User interaction with setup program 


\section{ACQUIR}

\subsection{GENERAL}

This program is the central part of the Doppler Ion Software system. It monitors the hardware, sends appropriate control commands to hardware, reads data from the Tracor Northern and writes the data to disk. In most instances, the program will run unattended once it is started up. The floppy disk w111 hold approximately 13 shots of data. When the disk is full, the screen is cleared, the teletype bell is rung and the program is suspended until an operator indicates that a new disk is in place.

For each shot on Doublet III, the program reads the shot number, sets up an interrupt register, reads the SETUP.DAT file, sends control information to the TRACOR, monitors the interrupt register to ascertain shot completion, gets the output from the TRACOR, and writes the spectral data and the setup data to disk. A complete description of the program interactions will appear in another section. Appropriate messages appear on the screen to indicate what is occurring, but no operator action is normally needed. The following diagram shows the file and hardware usage.

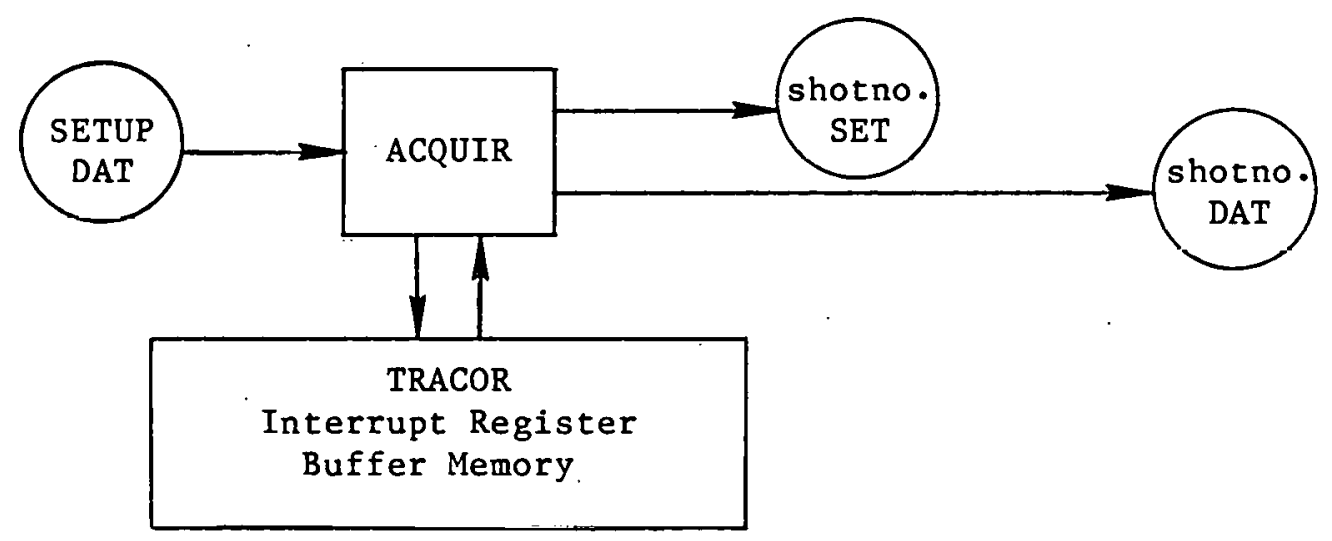


Operating in a standalone mode, the TRACOR can output the data to its own screen in about 2 minutes, 15 seconds ( 8196 data values). With the TRACOR talking to the LSI-11 through a buffer memory, output takes about 2 minutes, 25 seconds. Another 20 to 35 seconds is needed to write the data to disk and reset all parameters and hardware for the next shot. The entire cycle takes approximately 3 minutes. This allows the physicist 2 minutes (or more) to look at some of the data received. However, data can be analyzed at any later time, in an offline mode or on another LSI-11 computer.

Figure 3-1 shows screen output during normal execution.

\subsection{DETAILED ACQUISITION PROGRAM DESCRIPTION}

This section attempts to describe, in words, the steps and algorithms used in the program, and to note any problems encountered.

At the outset, it should be noted that the implementation of this program took several months due to all manner of delays. The TRACOR needed a wiring change to allow usage of the buffer memory. The 3608,3471 , and $3340 \mathrm{~B}$ all had hardware problems dropping bits. Various problems were found with the connectors between the 3340 and the TRACOR and the Tektronix 4010 and the LSI-11. Some problems occurred because the modules did not always easily slide into the backplane and make the proper connections. No one seemed to have any knowledge about exactly how the software should command the hardware and this was coupled with some rather sketchy manuals. The intermittent, early hardware problems made software development very challenging since one could not tell if software and/or hardware was/was not working.

Before the program is started, the TRACOR must be turned on, gain set, sequential scan on, scan advance on triggered, remote controller switch on RS232, I/O select switch on EIA, and the RS232 switch on. 


\section{R ACQUIR}

*****WARNING*****

SETUP INFORMATION IS OLD1ø/ 7/8ø 15: $\emptyset: 2$

****SHOT NUMBER BEING PROCESSED $=15050 . * * *$

STARTING OUTPUT 15:26: 7

STARTING OUTPUT 15:27:35

**DATA FILE OF 4\$96 POINTS FOR SHOT \# 15 $\$ 50$. WRITTEN ON DISK** *****WARNING*****

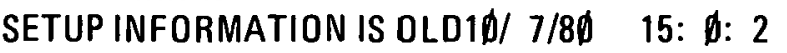

${ }^{* * * *}$ SHOT NUMBER BEING PROCESSED $=15 \emptyset 5 \emptyset . * * *$

Fig. 3-1. Sequential $\log$ during data acquisition 
The main routine, ACQUIR, controls all of the steps of the program. A standard CAMAC handler is used to interface to the equipment. Actions proceed in the following order:

1. Read SETUP.DAT file; get current date, time.

2. Send "on" command to TRACOR, ( $\langle$ CTRL Q〉, ASCII code 21) via CAMAC handler.

3. Clear and initialize 3340 buffer (see Kinetics Systems 3340/3340B hardware manual).

a. Via CAMAC - clear the output buffer.

b. Via CAMAC - write the mode register to enable end of block character detection, enable TTY control"signal, enable Data. Terminal Ready, enable Request to Send.

c. Via CAMAC - clear the LAM status register. Bits cleared are for - clear to send, data set ready, ring.indicator, EOB character decection enabied, output buffer empty.

4. Print message if setup file date is prior to current date.

5. Do status check of TRACOR to see if everything appears to be in proper configuration.

One point might be noted here. Commands can be sent to the TRACOR faster than it can respond. When the sequence gets "out of sync," the TRACOR appears to hang up. To avold this occurrence, frequent delays are done

a. Clear and initialize 3340 buffer (step 3 above).

b. Delay. 
c. Via CAMAC, send "C cr" to TRACOR. This should clear the TRACOR memory. After the command is executed, a "非" should be returned.

d. Via CAMAC, loop and test 3340B for bit 7 (input character available) to be set. Test is done 30000 times.

e. When input character is available, read the 3340 memory.

f. Test for first character equal to "非 sign. Status check is complete when this is true. If any of the first eight characters is a "非, repeat from step a. 4 times and go on to next step. If none of the first 8 characters is a "\#," print message and go on to next step.

6. Set the 9608 interrupt register.

The hardware is setup in the following way. The main control shot trigger, 7F, (6E with new operating system) will trigger the lst trigger in the Tooker trigger box. This Tooker trigger number 1 will set interrupt number 1 in the 9608. The last trigger in the Tooker trigger box sets interrupt number 2 in the 9608 .

The following functions are done (all via CAMAC):

a. Clear LAM and enable update.

b. Enable LAM.

c. Clear mask register.

d. Set mask register (interrupts $1 \& 2$ ).

e. Test if LAM enabled.

f. Test if update enabled.

g. Read the mask register and test for a "3" (interrupts $1 \& 2$ ).

h. Read the status register and test for 0 . 
7. Get the 24-bit shot number from the 3471. This number is controlled by a master computer and distributed to all the micro-crates which need it.

8. Set TRACOR control for entire memory.

a. Delay.

b. Send "G $1 / 1$ cr" to TRACOR. This will enable the entire memory. After the TRACOR has completed action, a "非 should be returned.

c. Read for 非. This little procedure will be repeated many times during program.

Read the 3340 LAM status register (up to 4000 times) and test for "input character available - bit 7." When character is available, read 1 character into memory from the 3340 .

d. Test returned character for "非" and print message if is not .

9. Clear the TRACOR memory.

a. Delay.

b. Send "C cr" to TRACOR. Clear the memory for Group $1 / 1$.

c. Read for \# (8c).

d. Test for 非 (8d).

10. Set up an. array to contain the proper ASCII to turn on the acquire light with the desired parameters. The command form is "A pl p2 p3 44 p5 p6" where values p1 through p6 are stored in the SETUP data file. They must be converted from binary to ASCII. 
11. Turn off the acquire light in case it is on.
a. Delay.
b. Send "A cr" to stop acquisition.
c. Read for 非 (8c).

12. Send the acquire command.

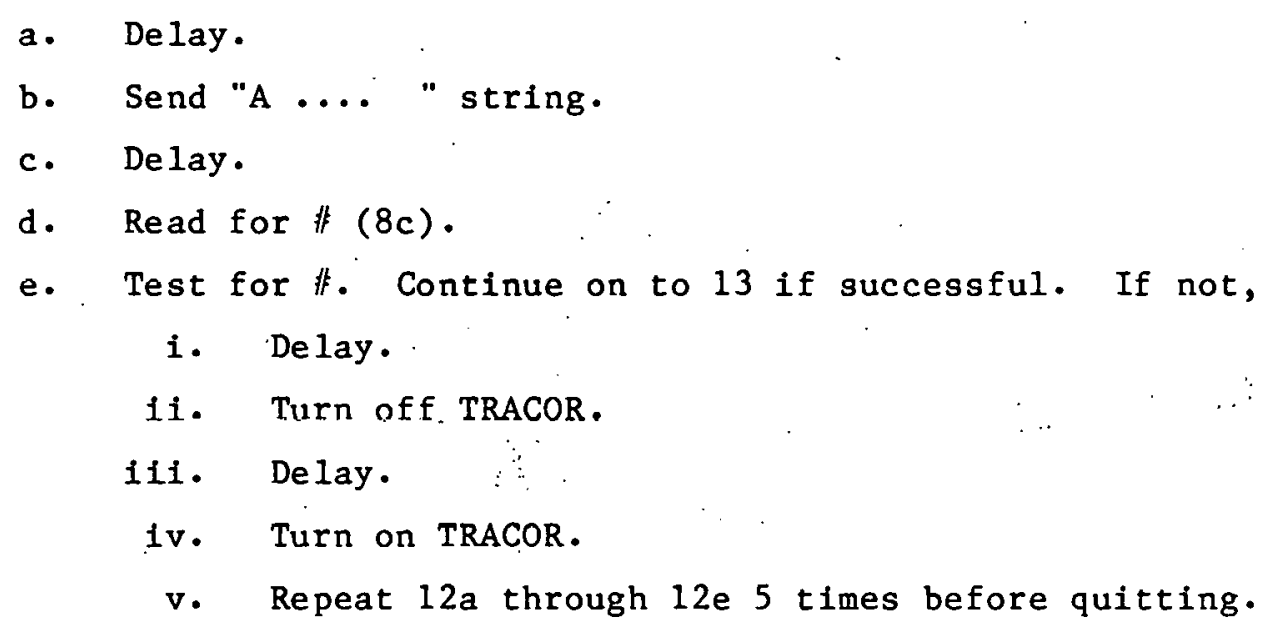

13. Setup arrays so that the current shot number will be used in the data 'file name.

14. Print message to screen. This message indicates that the hardware is in proper configuration for shot firing and that the program will be waiting for the $7 \mathrm{~F}$ trigger.

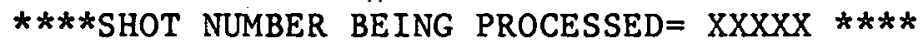

15. Test for shot being fired.

a. Read 9608 status register.

b. Test for interrupt 1 or interrupt 1 and 2 and go on to 16 when found. 
c. Delay 1 second and continue testing (go back to 15a).

16. Get the current time for this shot. This is. written in the file with the data.

17. Test for both interrupts in 9608 .

a. Read 9608 status register.

b. If both interrupts set, go on to 18 .

c. Delay $1 / 2$ second and repeat $17 a \& b$.

18. Calculate the number of points of data which should be acquired. This will be equal to the resolution times the number of triggers. If the total number is less than 8192, the TRACOR acquire light may not turn out and the TRACOR status command will return the number of sweeps rather than a .

19. Check the acquisition status.

a. Clear the 3340 buffer memory.

b. Send the status command " $5 \mathrm{a} . "$

c. Read 3340 LAM status register and check for character available (bit 7) (up to 4000 times).

d. Read 8 characters from 3340 buffer.

e. If these 8 are the same as expected according to 18 , then do the following:

i. Delay.

ii. Send "A cr" to turn out acquire light.

iii. Read for 非 (8c).

iv. Go on to 20 . 
If these 8 are not as expected:

i. Delay $1 / 2$ second.

ii. Try 19a-e, 20 times. After 20 times, print a message and get out of loop - do $19 \mathrm{e}$ i-iv.

20. Reset 9608 (same as all of 6).

21. Get the data in from the TRACOR. The TRACOR output command " 0 " results in ASCII lines of data being sent through the 3340 . The left side of the line contains a sequence number, then 8 data values of 7 characters each, and a carriage return, line feed at the right side of the line. The lines are 65 characters long, including the line feed. The data gathering scheme will entail... allowing one line of data at a time into the 3340 , checking that. the program sequence number and line sequence number are the same, checking that the data values are valid ASCII numerics, converting the numbers on the line to binary, cycling through all lines until all data is received. The 3340 buffer does not always stay exactly equal to the TRACOR's output and occasionally a line feed is lost. This is acceptable since the character is not crucial. It is for this reason, however, that the entire buffer memory of 128 characters in the 3340 is not used. The end of block character equal to "carriage return" has been set in the input endof-block switch 4 on the 3340 .

a. Clear 3340 (same as 3 ).

b. Get and display the current time. This informs the operator that data will be coming into the computer. 
c. Send the output command to the TRACOR and then find the first line of actual data (several header and title lines are also sent). The first line will start out (uniquely):

space space space space 0.0

i. Delay.

ii. Send "O sp 5 sp $0, "$ output EIA all data.

iii. Read 3340 LAM status register.

iv. Test for bit 2 on - end of block character detected (do iii and iv up to 60000 times).

v. If no EOF, go on to vii with an error code.

vi. As soon as $E O B$ detected in iv, write the mode register of the 3340 - value 648 - disable output EOB, enable input EOB, enable TTY, set data terminal not ready, disable request to send, enable input data.

vii. Read a line of output from 3340. Check that less than 2000 lines read. Read 3340 buffer.

v111. Clear 3340 buffer.

vx. Clear 3340 LAM status register (bit mask 358).

x. Write the 3340 mode register (bit mask 748). Enable input EOB character detection, enable TTY control, set data terminal ready, set request to send, enable input data. This will allow the next line of data to be transmitted into the 3340 buffer. 
xc. Read 3340 LAM status register and test for EOB sensed (bit 2 set to 1) (read and test up to 20000 times).

xii. Disable sending when EOB sensed. Write the mode register with mask $62_{8}$.

xiii. Read 3340 buffer (same as 2lc vii).

xiv. Look for the 7 starting characters in the contents read from the buffer memory. The first 30 lines sent from the TRACOR are scanned in sequence (i.e., repeat viii through xiv) and an error code set if not found. The location of the starting character of data in the buffer is kept. The code stops if no data is found.

d. Get the 8 (or fewer) data items in the current line. Scan for a \# which would indicate no more data. Verify that each character is an ASCII numerical. Store as binary in data array.

Get the next line of data into memory.

i. Clear 3340 buffer.

ii. Clear 3340 LAM status register (mask $35_{8}$ ).

iii. Write 3340 mode register (mask 748 ).

iv. Read LAM status register and test for End of Block bit set.

v. Stop transmission when end of block sensed (write mode register mask 628). 
vii. $\operatorname{Read} 3340$ line (21c vii).

viii. Check line for a '非 or ' .' and note location.

f. Repeat d \& e until all data are collected. When finished, get current time and display.

g. Clear 3340 (same as 3 ).

22. Write the data to disk - 2 files:

shotno.SET and shotno.DAT

If the disk is full, the screen will clear, the bell will ring and the program will pause for the disk to be replaced and the program continued. The program can potentially hang indefinitely here if no one is around to change the disk.

23. Prepare for next shot. Restart at 3 . 


\section{PROGRAM PLTDAT}

\subsection{GENERAL}

The analysis program, which consists of many types of plots and some calculations, is written to interact with a physicist who is familiar with the Doppler Ion temperature diagnostic. The program is user-interactive and will not run in a stand-alone mode. The physicist must make choices about which peaks to examine and about straight line fits in order to arrive at accurate calculations of ion temperatures.

This document will not attempt to describe all of the theory concerning the calculations. The calculations which are made will be noted.

The actual program requires a large amount of core and is overlayed so that it will fit into memory. Processing is very rapid despite the overlays. The plot routines used are simple and assume the calling routines have set up data in a reasonable manner. The plot routines do not account for dark lines or plot values outside the defined window area. A separate report documents the actual plot package used GA-A16148.

\subsection{PROGRAM OPERATION}

Program execution is initiated by typing $R$ PLTDAT. After the desired shot number is entered, the data file and setup information are read and a short summary of the lnformation is displayed. The first option is to plot all of the spectra. The operator must enter a 'Y' or 'N.' At any time later in code execution, if the operator enters ' $R$,' the program will return to the point of 'PLOT ALL SPECTRA.' 
R PLTDAT

ENTER SHOT NUMBER TO PROCESS OR BLANK

14048

14048. $8 / 5 / 8 \emptyset \quad 2 \emptyset: 5: 3 \quad$ NPTS $=8192 \quad \mathrm{ET}=\emptyset .50 \phi \emptyset \phi \emptyset 0 \mathrm{E}-\emptyset 1$

8 TRIGGERS $=\quad \emptyset \quad 90 \quad 19 \emptyset 29 \emptyset 39 \emptyset \quad 49 \emptyset 59 \emptyset 69 \emptyset$

PLOT ALL THE SPECTRA-Y OR N

$\underline{Y}$

Inputs from the operator will be underlined.

All of the spectral data will be plotted, 2 graphs per page. See Figs. 4-1 through 4-4. The user must enter 'return' to continue to next page. No automatic hard copy call is made, but the user may control this operation. The plots of all spectra will be on the same scale which reflects the max and min values of all data. The points which are plotted are the average of each adjoining set of 4 points.

After all spectra are plotted or if ' $N$ ' was entered above, a particular spectrum can be chosen. This message will repeat if an inappropriate number is entered. This plot is also a 4 point-averaged curve, but the scale reflects only this spectrum.

\footnotetext{
ENTER SPECTRUM NUMBER TO SEE-1 TO 16 $\underline{2}$
}

The next question is 'IDENTIFY PEAKS?' This option allows the user to use the crosshairs on the Tektronix 4010 to locate the approximate channel number of a peak, and obtain the peak value at this point. The crosshair cursor can be moved to the appropriate position (only the horizontal value is used) and then a printing character ( $i . e ., A, B, X$ ) followed by return entered. The $X, Y$ values of the peak are listed and then another peak search may be done. When done with the peak search, $a$ ' $N$ ' must be entered. 
2ø:5: 3 ET $=5 \emptyset$ MSEC

G MSEC SPECTRUM $=1$ C IV 1543.3A CALIBRATION

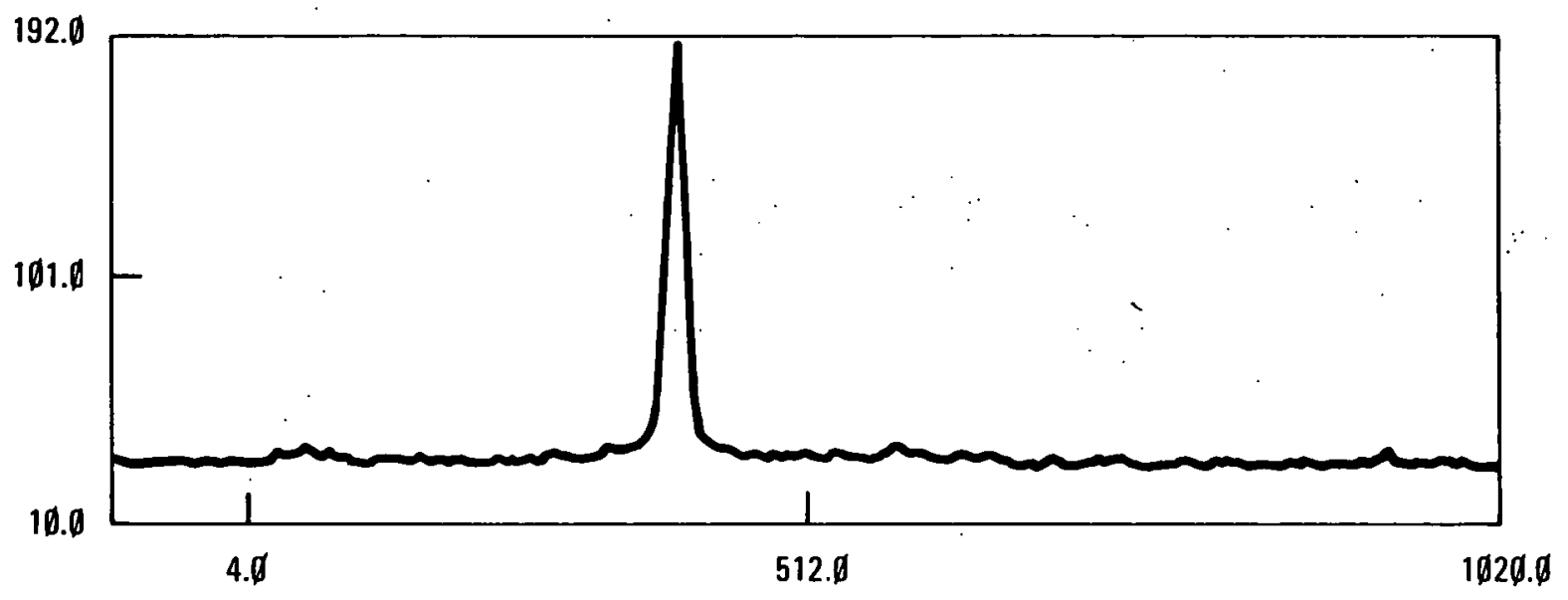

90 MSEC SPECTRUM $=2$

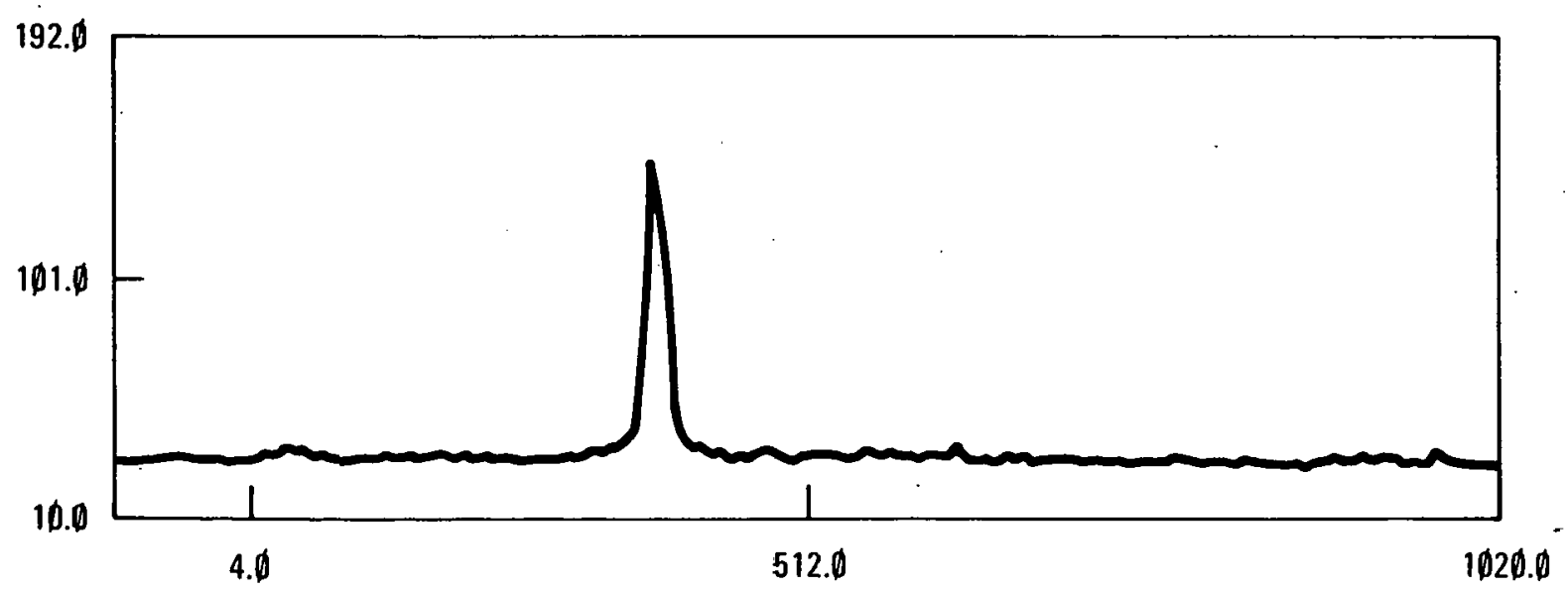

Fig. 4-1. Raw spectral output 
DOPPLER ION

14048. $8 / 5 / 8 \emptyset \quad 20: 5: 3 \quad E T=50$ MSEC

190 MSEC SPECTRUM $=3$ C IV 1543.3A CALIBRATION

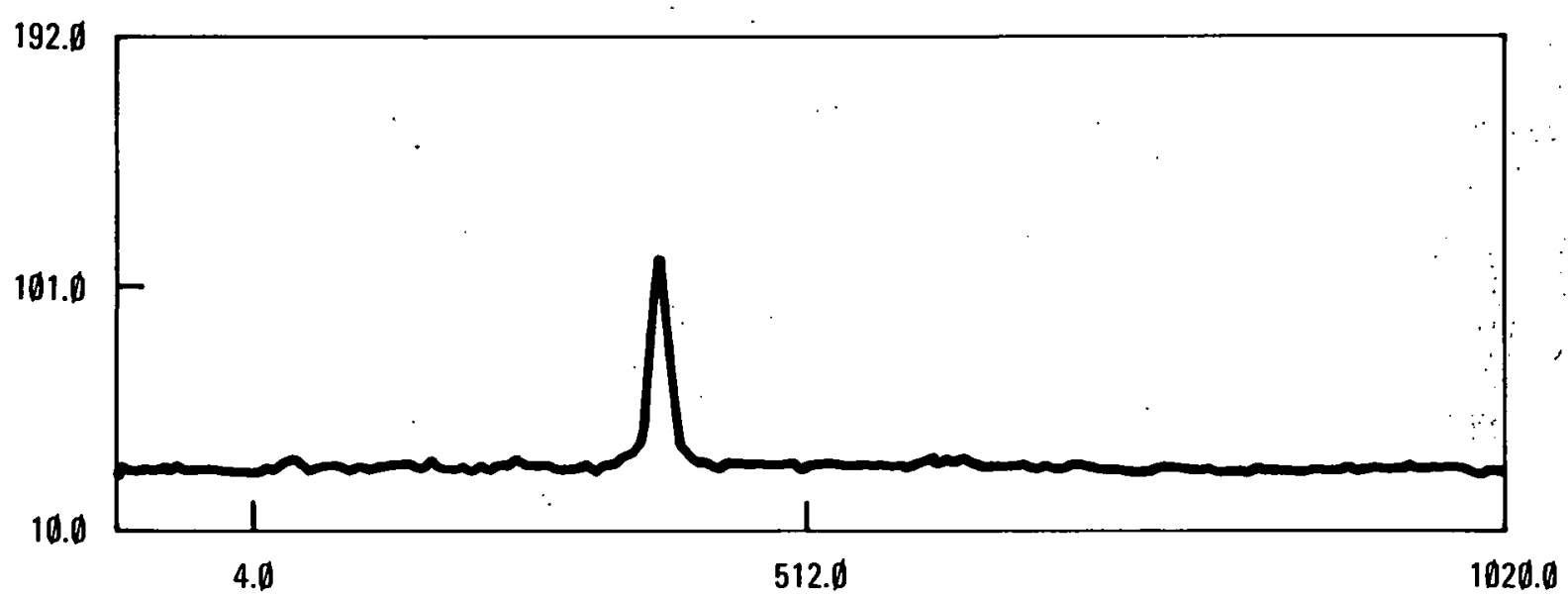

N

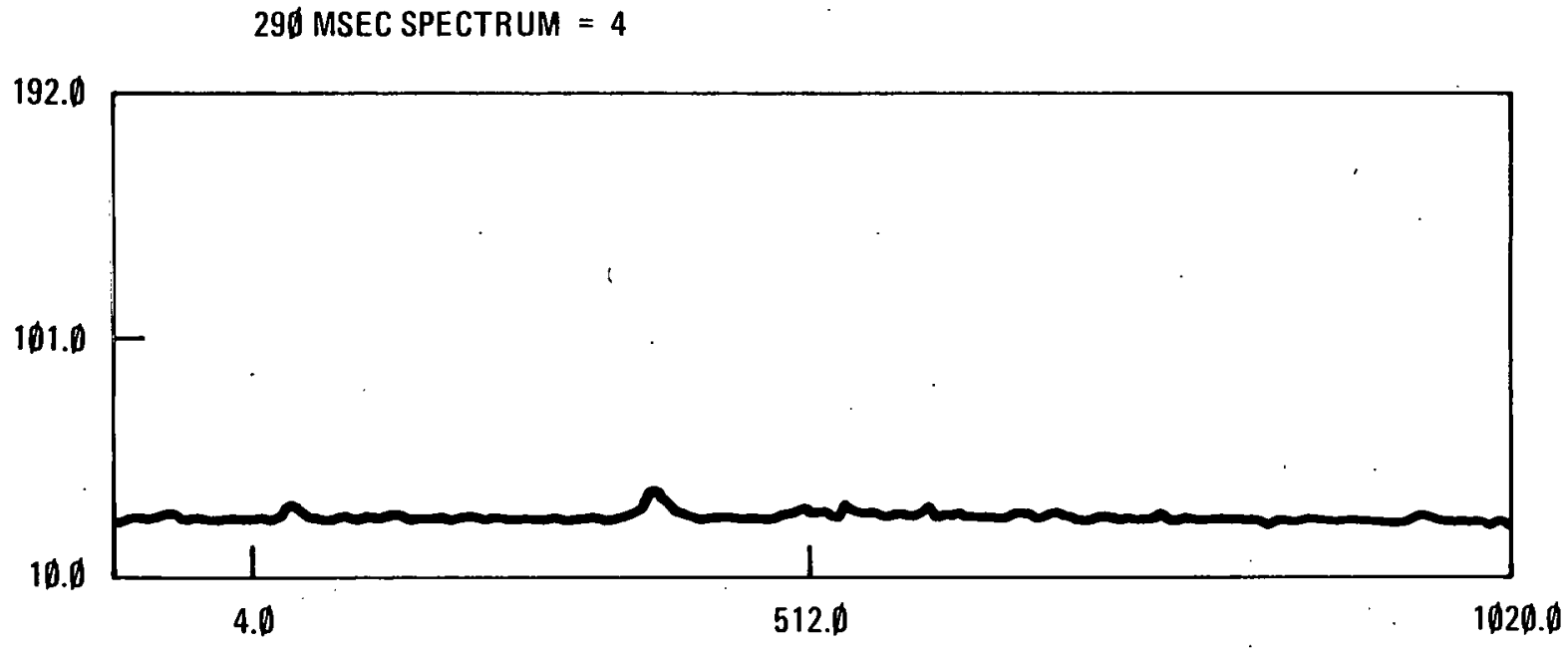

Fig. 4-2. Raw spectral output 
DOPPLER ION

140<8. 8/5/80 20:5: $3 \quad$ ET $=50$ MSEC

39ø MSEC SPECTRUM $=5$ C IV 1543.3A CALIBRATION

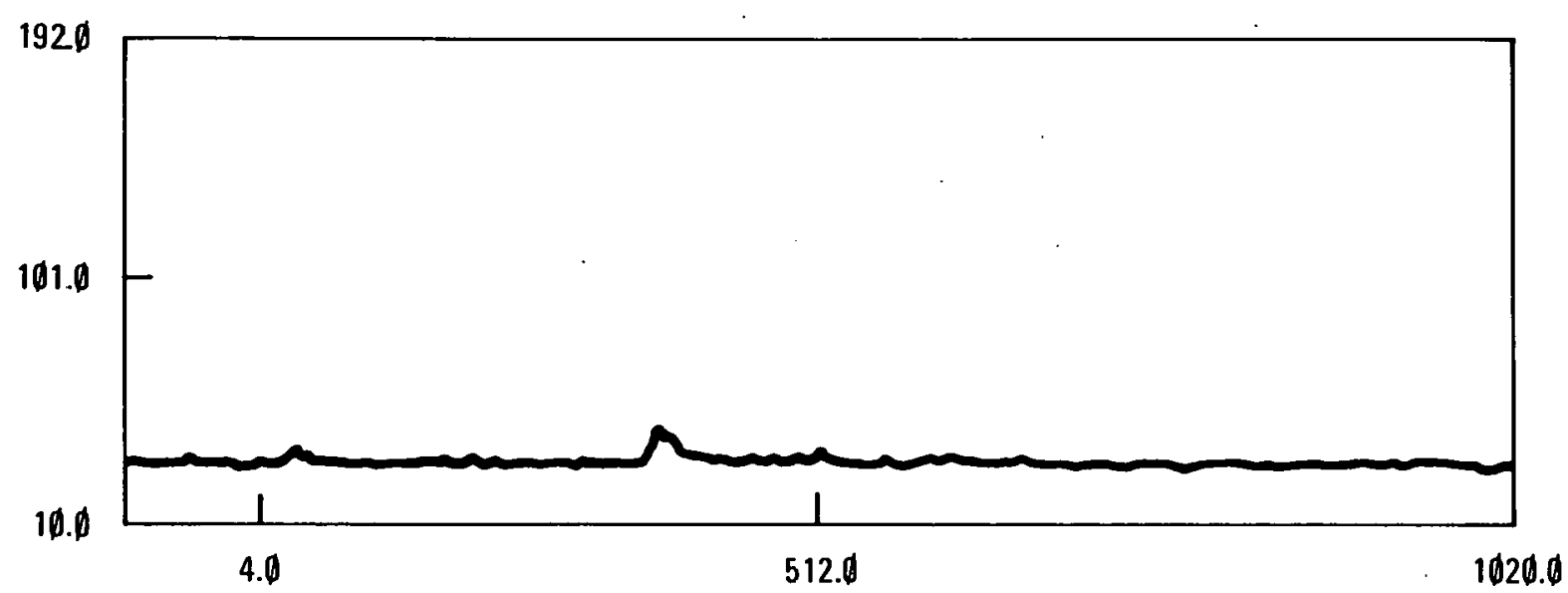

49D MSEC SPECTRUM $=6$

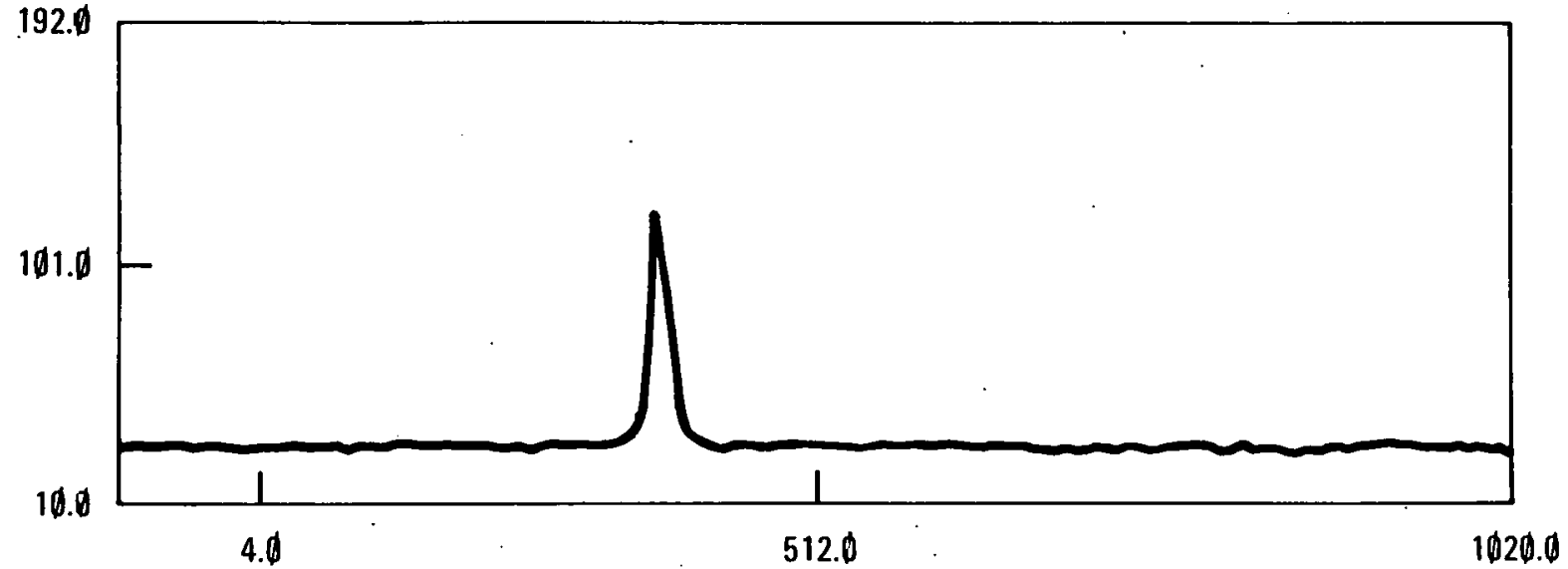

Fig. 4-3. Raw spectral output 
DOPPLER PON

14048. $8 / 5 / 80 \quad 20: 5: 3 \quad$ ET $=50$ MSEC

590 MSEC SPECTRUM $=7$ C IV 1543.3A CALIBRATION

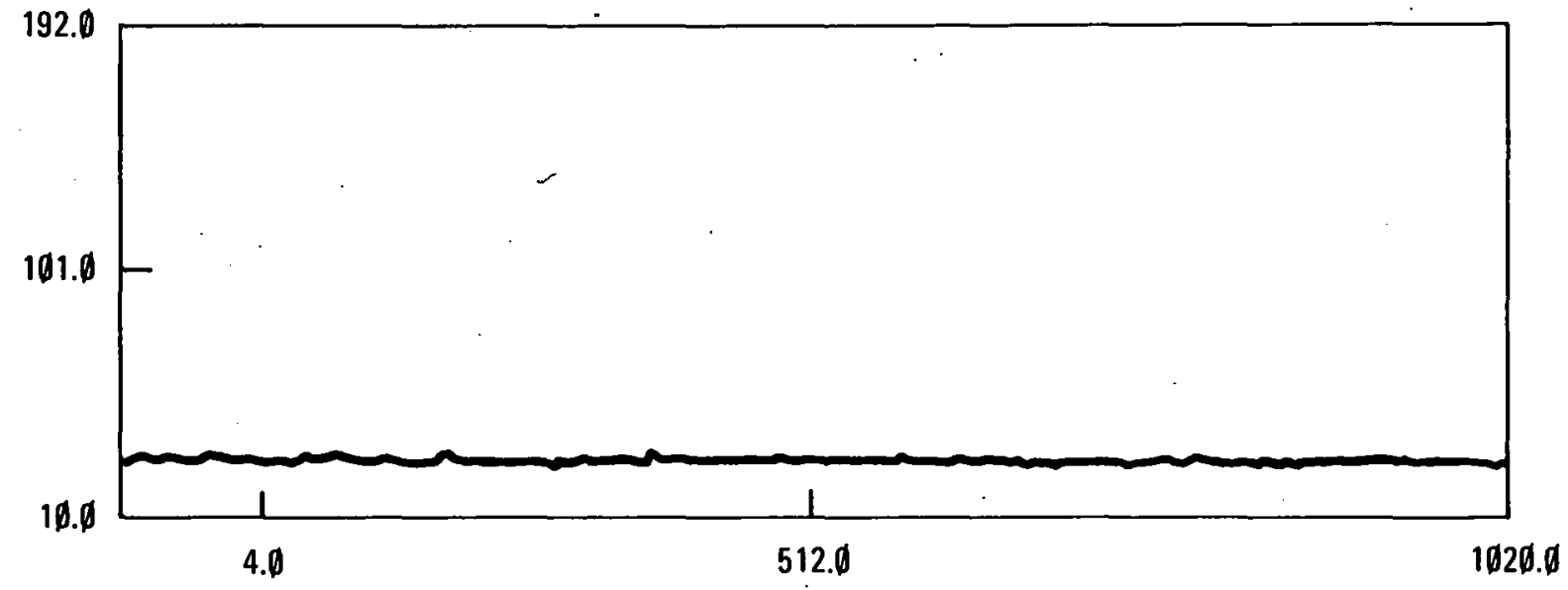

$\stackrel{N}{\infty}$

$69 \emptyset$ MSEC SPECTRUM $=8$

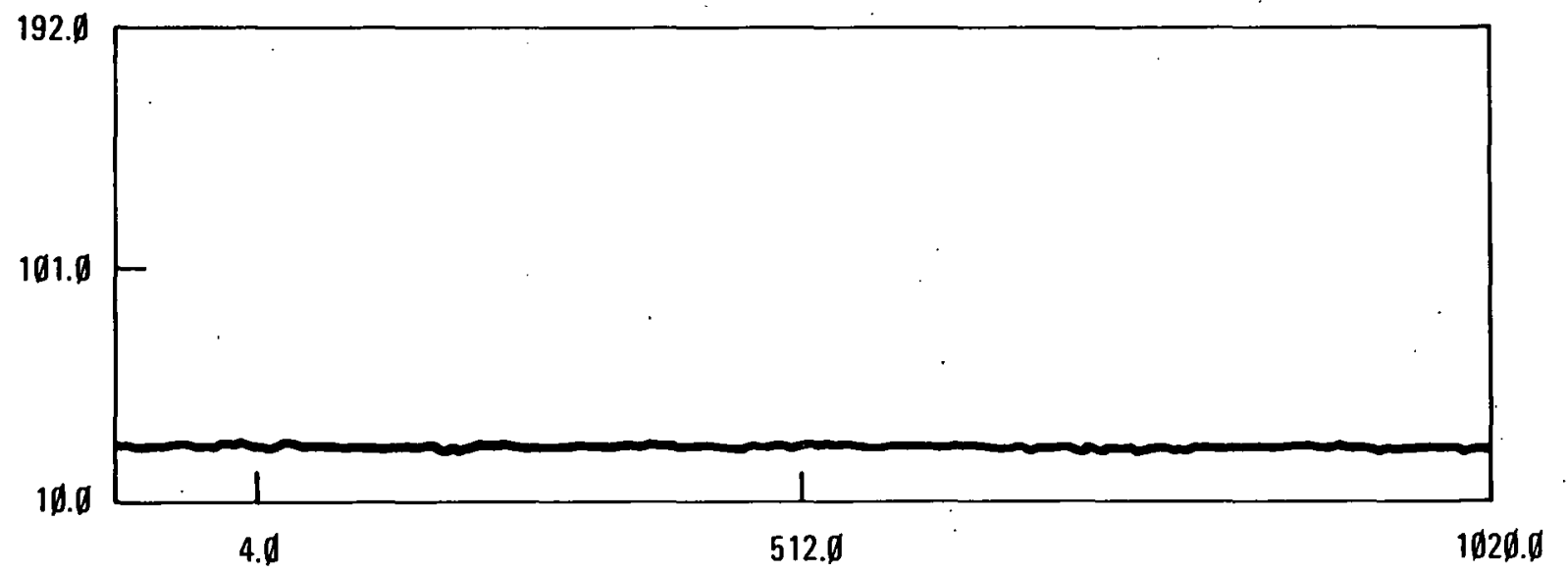

Fig. 4-4. Raw spectral output 


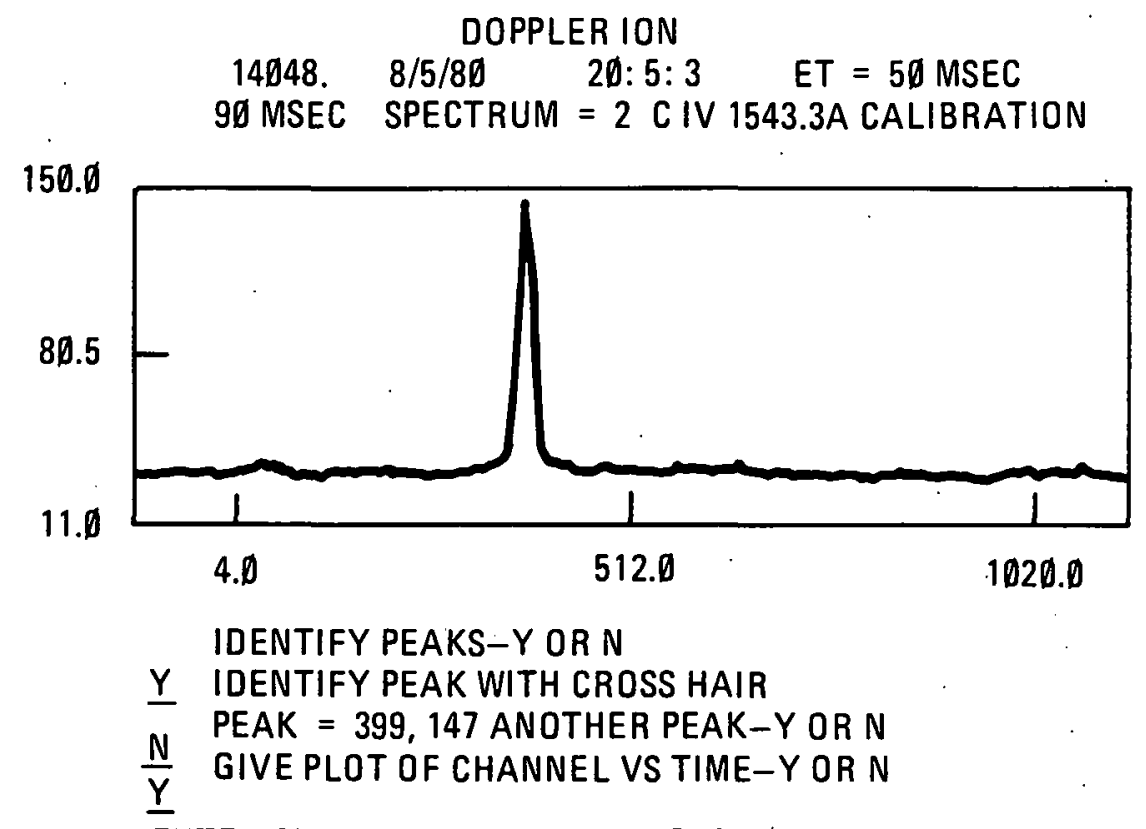

ENTER CHANNEL NUMBER FOR PLOT

$\underline{399}$

If a peak search has been done, the user will have the option to plot a particular channel vs time. If this option is chosen, the user indicates the desired channel (see above). The screen automatically clears, and a plot of intensity vs time for this one chosen channel is done. It is very probable that the desired peak will move a small amount with time. An option is given to the operator to do a 'PEAK SEARCH.'

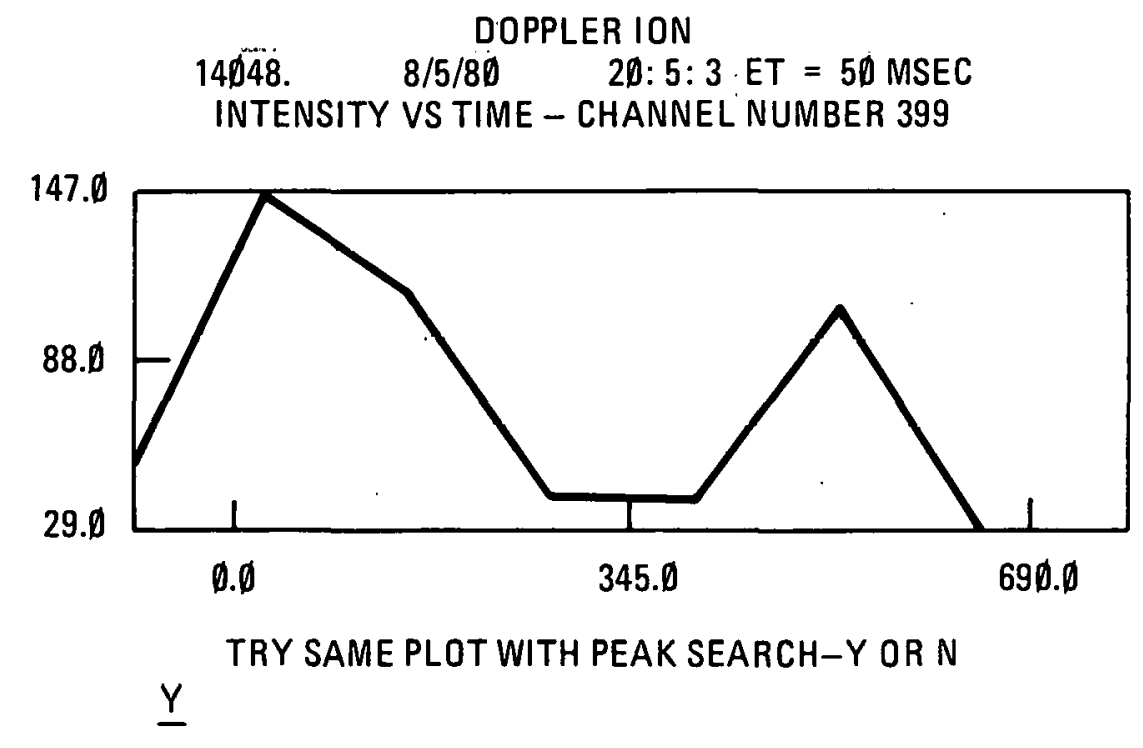


The peak search examines points 5 to the left and right of the designated channel, then 10 , then 15 , then $20,25,30,35$, and 40 , looking for a local maximum. If no $\max$ is found, the value of the chosen channel is used. This plot is labeled 'ADJUSTED CHANNEL.' A return must be entered for the program to continue.

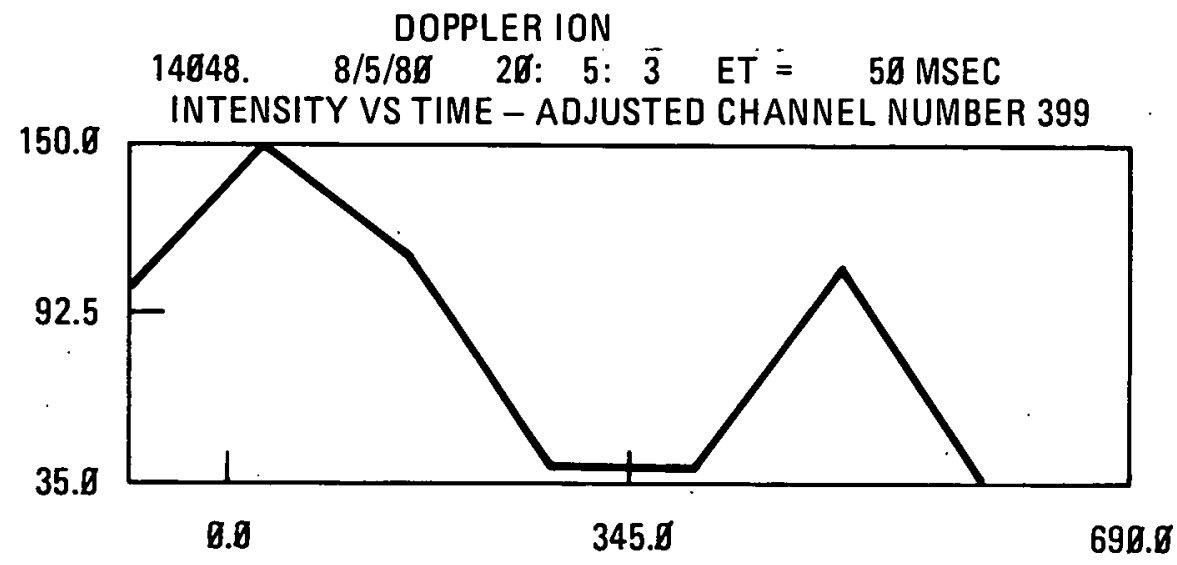

At this point, the user will again be asked to chose a spectrum and it will be plotted on the top half of the screen (averaged values are plotted). If peak identification is not chosen, the crosshair will appear again with the message 'EXPAND REGION.' See Fig.-4-5. Only the horizontal component of the crosshair is needed, and after the first is entered (a character followed by return), the crosshalr will automatically reappear and the second value can be entered. The values can be entered in any order. However, if the number of channels encompassed is greater than 128 , the values must be reentered to lie within this limit. The plot of the expanded region gives every point.

The user must now decide on whether to substract the background information. This is relevant only if the hardware is set up appropriately. The program assumes that spectrum number 1 is background, and each channel from spectrum 1 will be subtracted from the same channel of the spectrum currently being viewed. Any resulting value less than 1 is set to 1 . Once this subtraction has been done, all further use of data for this spectrum will be with the adjusted data. The actual data on the disk will not be altered. A plot of the adjusted data is given if background subtraction is selected. 


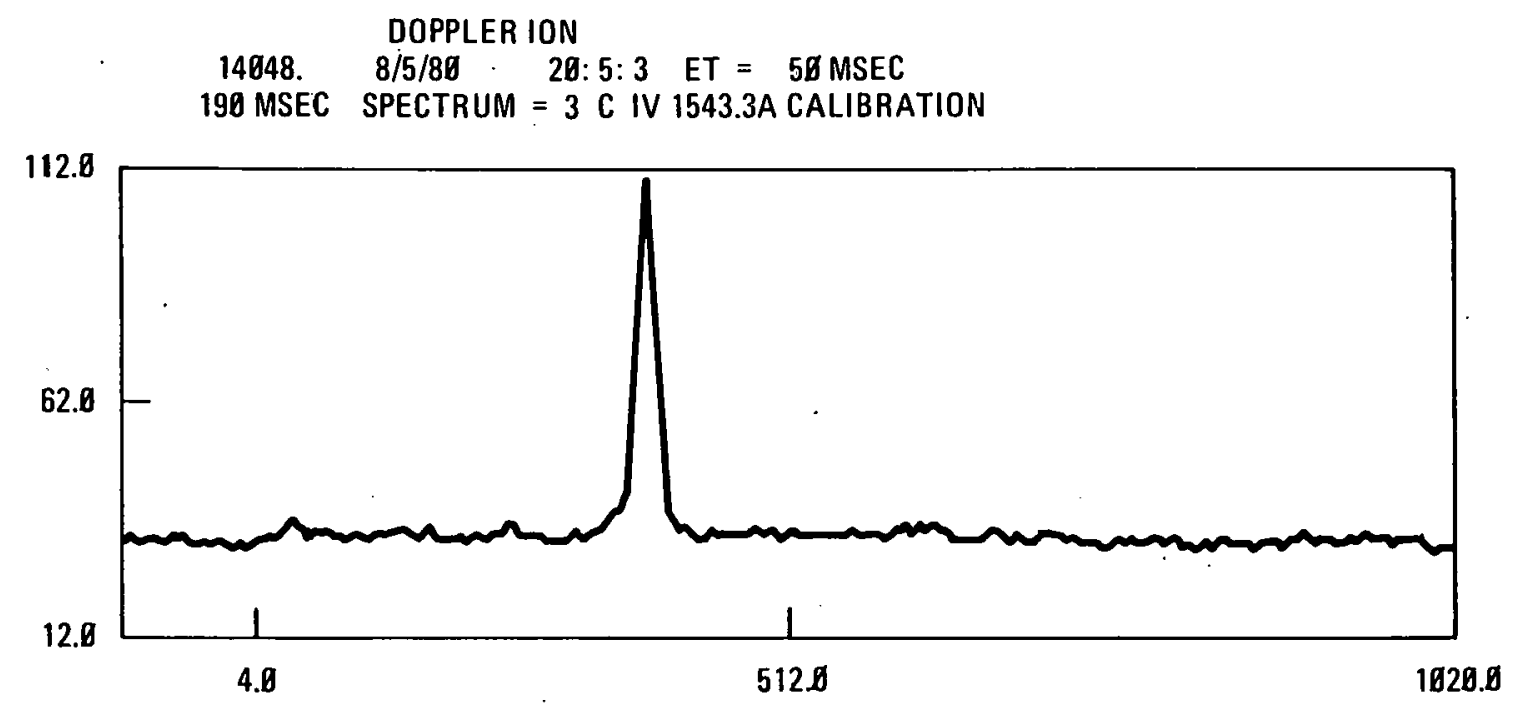

IDENTIFY PEAKS-Y OR N EXPAND REGION WITH CROSSHAIRS

N

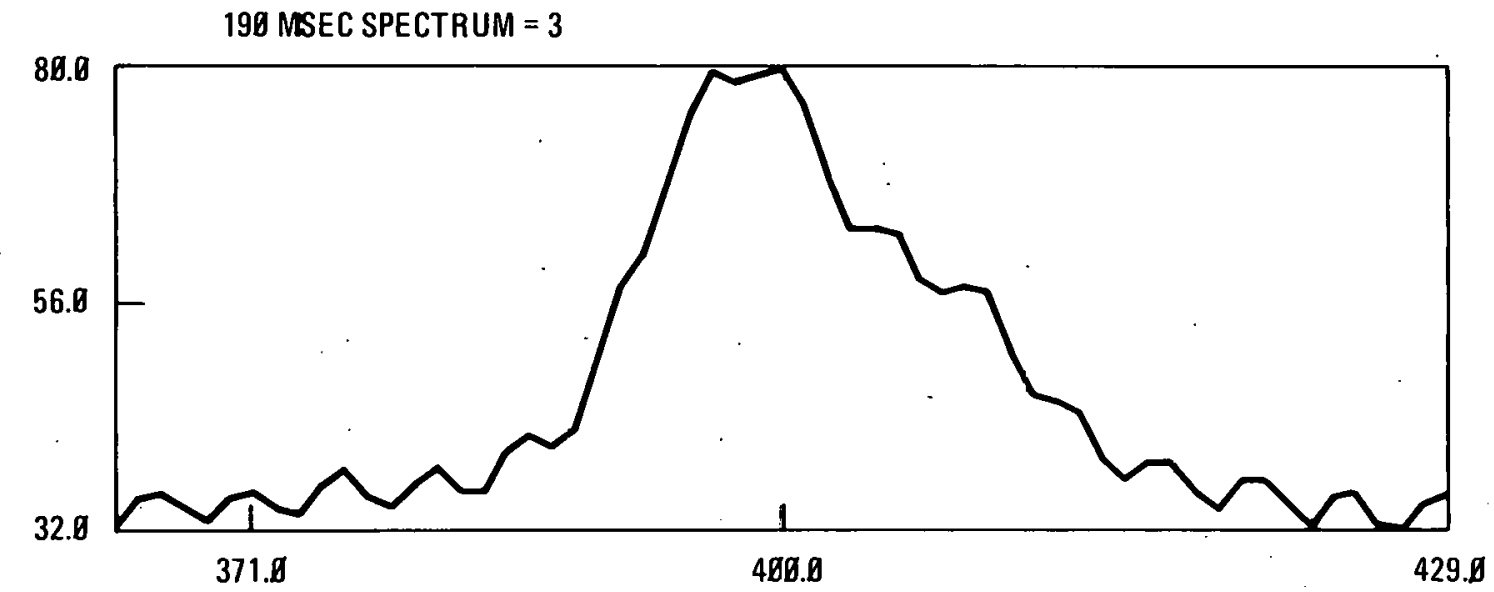

SPECTRA MAX AT 4פø, 112 SUBTRACT BACKGROUND-Y OR N

Fig. 4-5. Raw spectral data and a selected subset 
The next option is to smooth the data. If chosen, all channels of data for the given spectrum are smoothed and the resulting values are plotted. Smoothing can be executed multiple times, although 2 or 3 are usually sufficient. The data used for this spectrum will be in the smoothed form for the rest of program execution although the disk data is not affected. The same algorithm is used as exists internally in the TRACOR.

$$
y^{\prime}(n)=\frac{y(n-1)+2 y(n)+y(n+1)}{4}
$$

See Fig. 4-6 to see the results of several smoothings.

After the smoothing is complete (this includes not smoothing), four options are presented to the user (see below).

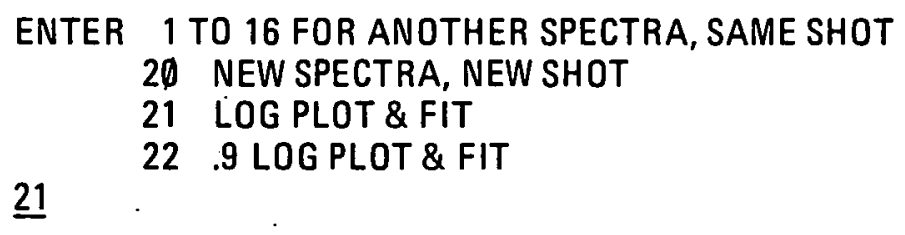

If a number 1 to 16 is entered (assuming it is a valid spectrum), the program cycles back to plot one spectrum and ask for peak identification. If a 20 is entered, the user will be asked for a new shot number and restart at the very beginning of the program.

In order to compute an ion temperature, 21 or 22 must be entered. Figure 4-7 shows a plot of 2 curves and one straight line which resulted after entering 21. The subsection of the current spectrum is used to generate the graph. $\lambda_{0}$ is the channel number which corresponds to the maximum intensity in the chosen subregion. This choice is made from the data in its most current form, i.e., with background subtracted, with smoothing or whatever combination has been done with this particular spectrum. The 2 curves are not necessarily the same length since the chosen subregion is probably not symmetrical around $\lambda_{0}$. The 2 curves are computed as

$$
\begin{aligned}
& y(j)=\ln \left(I_{j}\right) \\
& x(j)=\left(\lambda_{0}-j\right)^{2}
\end{aligned} \quad j=\text { channel numbers in subregion }
$$


8/5/86 26:5:3 ET $=58$ MSEC

190 NSEC SPECTRUM $=3$ C IV 1543.3A CALIBRATION

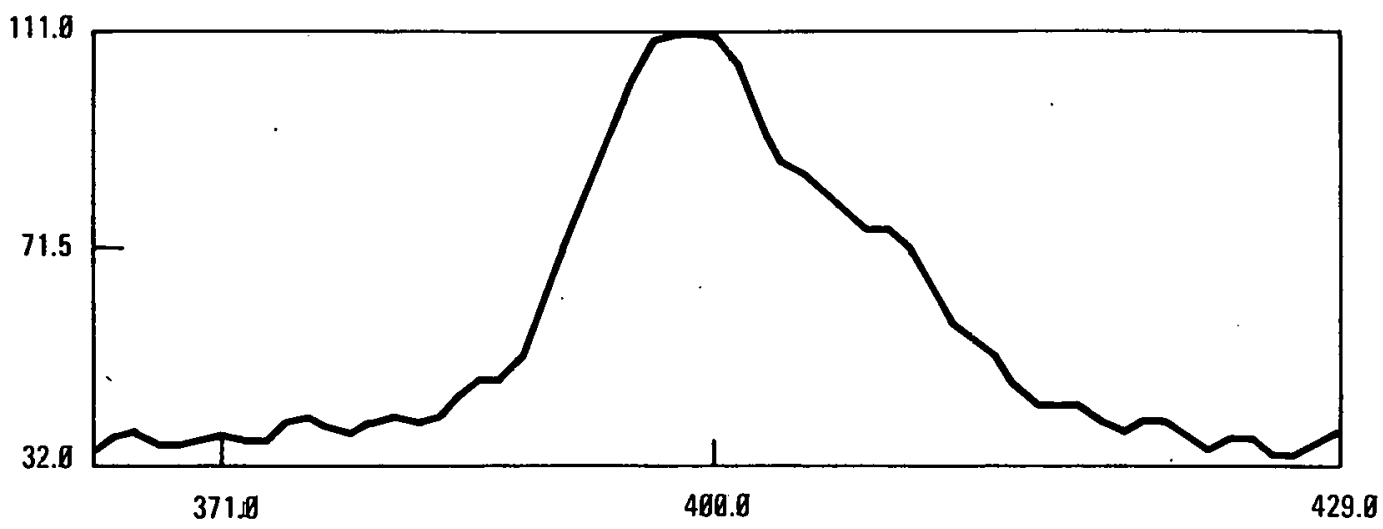

SMOOTH-Y OR N

14948. $8 / 5 / 80 \quad 29: 5: 3 \quad \mathrm{ET}=50 \mathrm{MSEC}$

190 MSEC SPECTRUM $=3$ C IV 1543.3A CALIBRATION

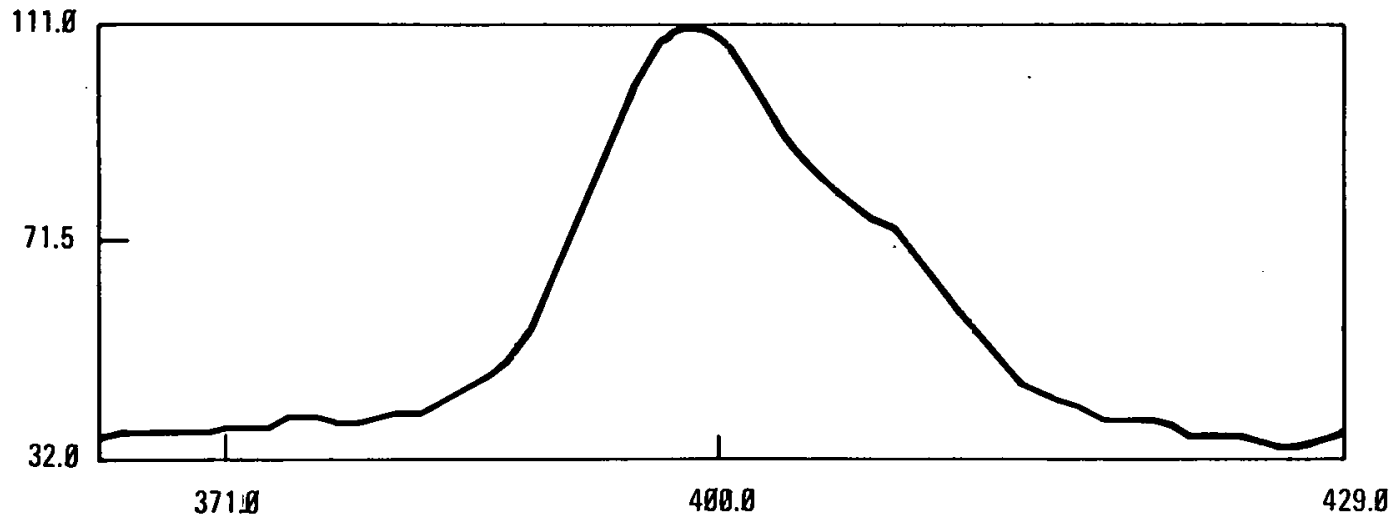

SMOOTH-Y OR N

Fig. 4-6. Two iterations of smooth data 
DOPPLER ION

14848. 8/5/80 28: $5: 3$ ET = 50 MSEC

190 MSEC SPECTRUM $=3$ C IV 1543.3A CALIBRATION

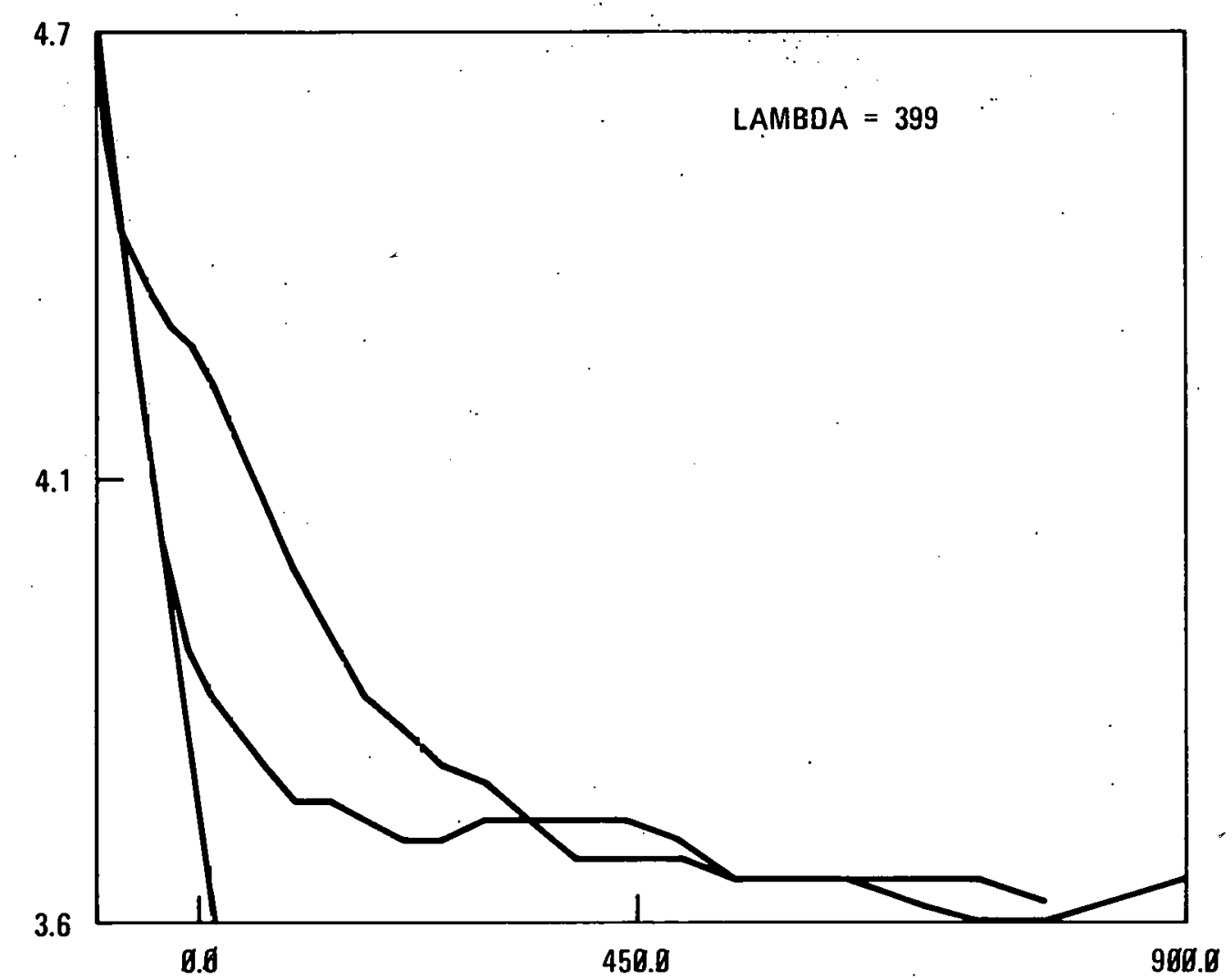

DO FIT-Y OR N

Y TRY ANOTHER FIT-Y OR N

$\overline{\mathrm{N}} \quad$ DELTA LAMBDA**2 VS LN(I)

ENTER STRAIGHT LINE FOR FITI USING CURSOR

F-g. 4-7. Fitted data 
Lambda is the value of $\lambda_{0}$ used for the expansion.

The user can decide if a fit is justified at this point and enter ' $Y$ ' or ' $\mathrm{N}$ ' to the question 'DOFIT.' If a fit is chosen, the user will have multiple trys at entering a straight line on the graph (which has only 2 curves at this point). The crosshairs will appear and 2 locations can be indicated. If the resulting straight line is satisfactory, the user should enter ' $N$ ' to indicate the last' entered line is acceptable.

Most information necesary for the calculation is already present. However, the order number, the atomic number corresponding to the peak chosen and lambda must be entered once. Once entered, it will be returned and does not have to be retyped.

\section{WISH TO ENTER ORDER \#, ATOMIC \#, ETC-Y OR N \\ Y \\ ENTER ORDER \#, ATOMIC \# AND LAMBDA \\ 8121543}

The graph displayed in Fig. 4-8 will be displayed with the final calculated results. A straight line interpolation value is computed from the dispersion table based on the spectrometer wavelength setting. Similarly, a straight line interpolation is used in the FWHM table based on the mid-point time the spectrum occurred (i.e., trigger time for this: spectrum $+1 / 2$ ET). Appropriate end points are used for values outside the table ranges (no extrapolation is done).

The program uses the following numbers in its computation. If $y_{1}$ and $y_{2}=y_{1}-0.69$ are values on the straight line fit, the value $x 69$ is computed from the corresponding $x_{2}$ and $x_{1}$ coordinates $\left(x_{69}=x_{2}-x_{1}\right)$.

The actual ion temperature is calculated as

$1.7 \mathrm{E} 8 *$ a tomic number $*\left[\frac{\left(\frac{\text { Dispersion }}{\text { order }}\right)^{2} * \times 69}{(\text { lambda entered })^{2}}-\left(\frac{\text { FWHM * Dispersion }}{\text { lambda * order }}\right)^{2}\right]$ 


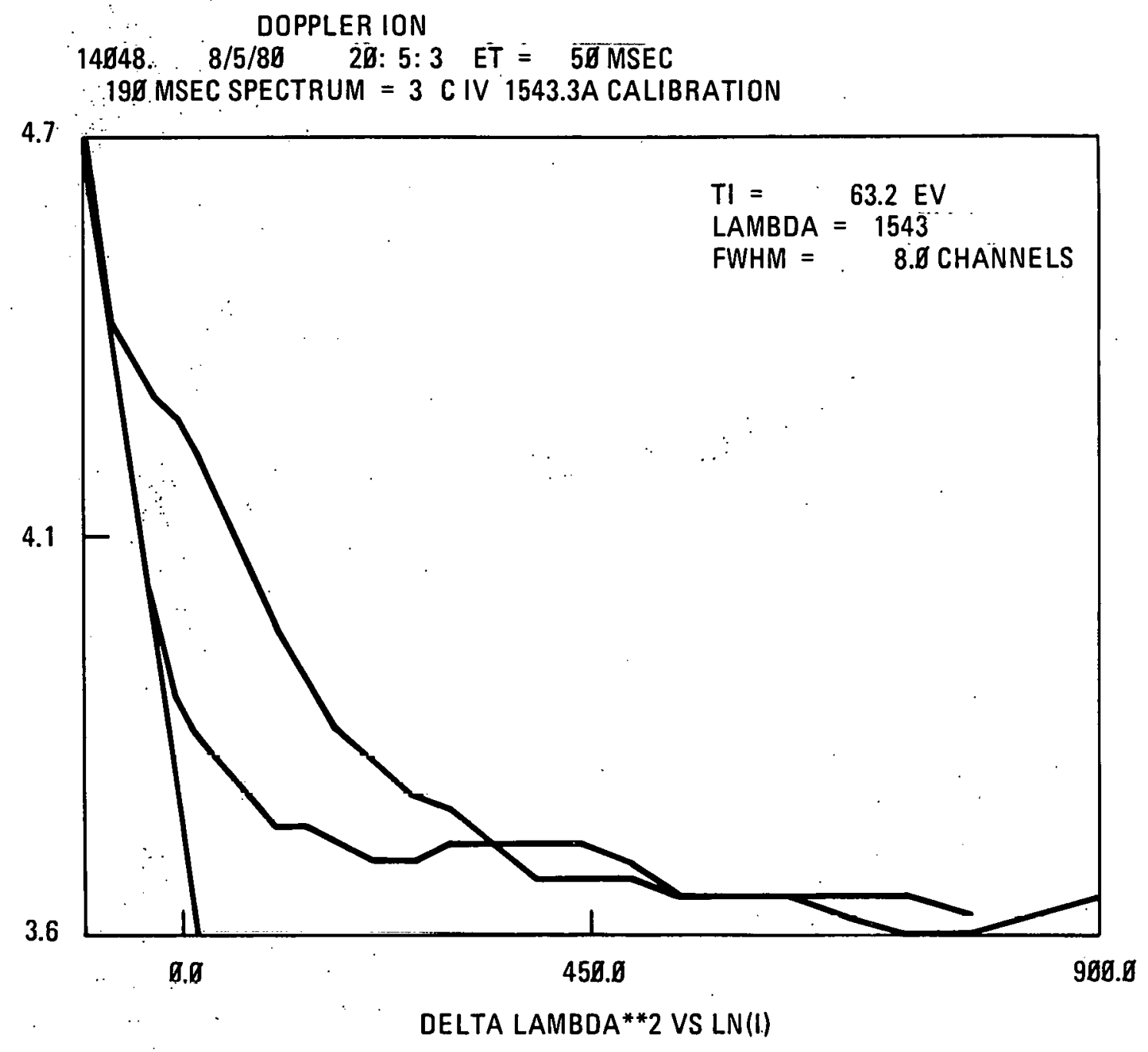

Fig. 4-8. Fitted data 

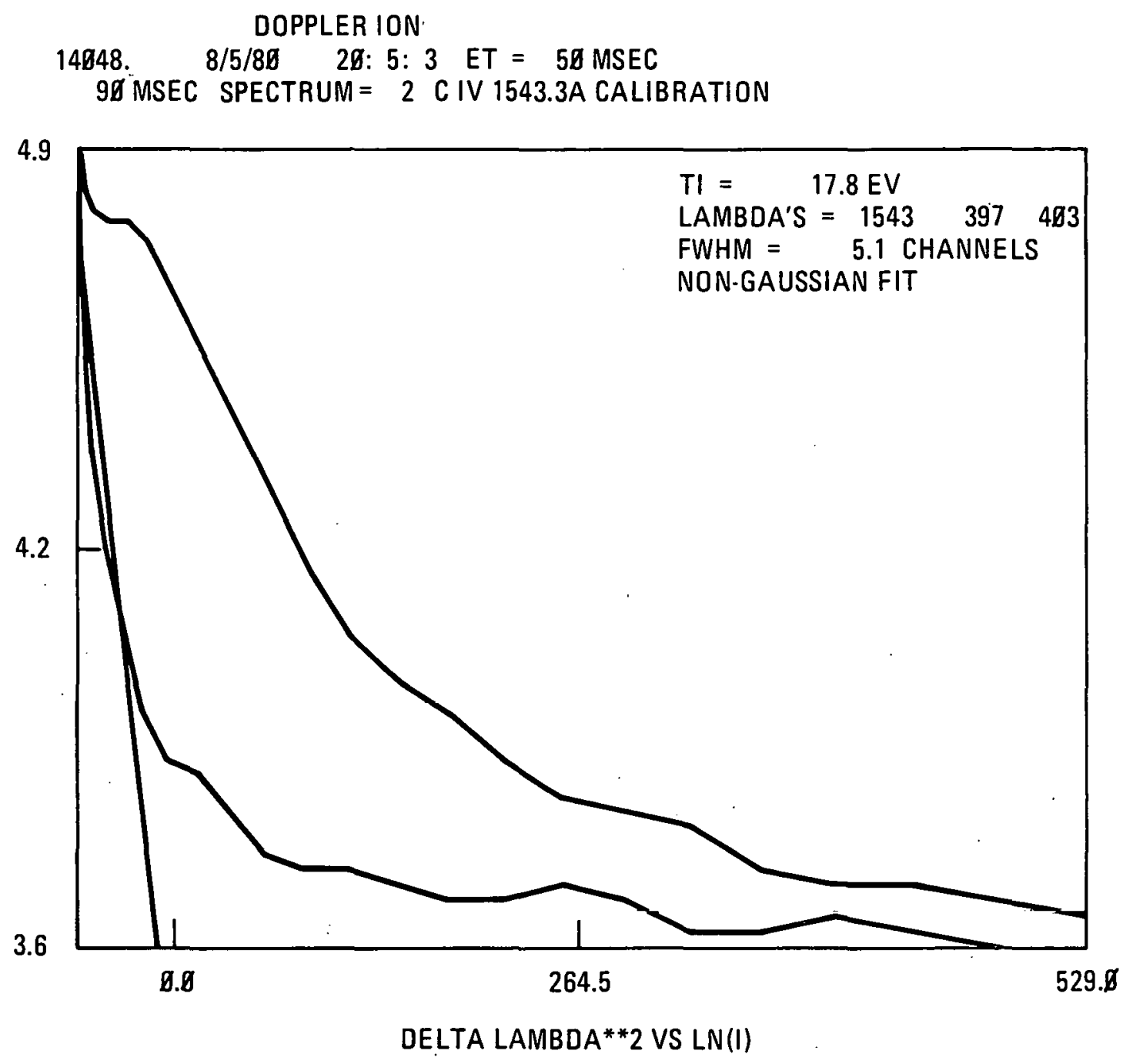

Fig. 4-9. Fitted non-gaussian data 
See Appendix A for a description of the calculation. This is from GA-1595, 6/13/80 by John Lohr.

All numbers needed for FWHM are at hand. This value is

$$
\text { abs }\left[\left(\lambda_{0}-\sqrt{x}_{1}\right)-\left(\lambda_{0}-\sqrt{x}_{2}\right)\right]
$$

After the plot is completed, the user again has choices of another spectrum, new shot, $\log$ plot or $0.9 \mathrm{log}$ plot. The $0.9 \mathrm{log}$ plot and fit (code 22) is identical to the normal fit except that the expansion is done around $\lambda_{1}$ and $\lambda_{2}$ where

$$
\begin{aligned}
& \text { I }\left(\lambda_{1}\right)=0.9 \text { I }\left(\lambda_{0}\right) \\
& \text { I }\left(\lambda_{2}\right)=0.9 \text { I }\left(\lambda_{0}\right)
\end{aligned}
$$

$\lambda_{0}$ is the same definition as above. This fit is labeled Non-Gaussian and in addition to lambda, the two channel numbers used for the expansion are 1isted. See Fig. 4-9. . 


\section{APPENDIX A *}

For the thermal distribution function with negligible collisional broadening the Doppler shift for emitting ions results in a Gaussian line shape

$$
I_{D}(\Delta \lambda)=\left[\frac{M_{i} c^{2}}{2 \pi k T_{i} \lambda_{0}^{2}}\right]^{1 / 2} \exp \left[-\frac{M_{i} c^{2}}{2 k T_{i} \lambda_{o}^{2}}(\Delta \lambda)^{2}\right]
$$

where the normalization gives $\int I_{0}(\Delta \lambda) d \lambda=1, M_{i}$ is the ion mass, $\lambda_{0}$ is the line center wavelength and $\Delta \lambda=\lambda-\lambda_{0}$.

The FWHM for this distribution is

$$
\Delta \lambda_{D}=\left[\frac{2[\ln (2)] k T_{i}}{M_{i} c^{2}}\right]^{1 / 2} \lambda_{0}
$$

so that

$$
\mathrm{k} \mathrm{T}_{\mathrm{i}}(\mathrm{eV})=1.7 \times 10^{8}\left(\frac{\Delta \lambda_{\mathrm{D}}}{\lambda_{\mathrm{O}}}\right)^{2} \mathrm{~A}
$$

where $A$ is the atomic mass number of the emitting ion, and $\Delta \lambda_{D}$ now denotes the Doppler FWHM for the line.

*From private conversation with John Lohr, 6/13/80. 
In the absence of any Doppler shifts instrumental effects will produce a broadened Gaussian-like line profile for a monochromatic source. This results in an "instrumental temperature" which must be unfolded from the measured value. Therefore the ion temperature can be determined from

$$
k T_{i}(e V)=1.7 \times 10^{8}\left[\left(\frac{\Delta \lambda_{D}}{\lambda_{0}}\right)^{2}-\left(\frac{\Delta \lambda_{\text {inst }}}{\lambda_{0}}\right)^{2}\right] \mathrm{A}
$$

where $\Delta \lambda_{\text {inst }}$ is the instrumental broadening, in channels, times the dispersion for the order in question, in $\AA /$ channel.

For the magnetic and electric fields typically encountered in Doublet III Stark broadening is negligible. Zeeman splitting gives $\Delta \omega \cong 1.8 \times 10^{11 /}$ sec, which gives a wavelength shift of about $\pm 0.1 \AA$. This is a small, but non-negligible, effect. In practice, what appear to be Zeeman-distorted line shapes can be adjusted subjectively during analysis. 


\author{
APPENDIX B \\ TN-1710-31, REMOTE CONTROL INTERFACE
}

The TN-1710-31 is a universal remote interface which allows an external device to manipulate most of the TN-1710 front panel functions. The controlling device may be either a Teletype or equivalent, a Computer, Acoustic Coupler, or Programmable Calculator. This module operates in conjunction with the standard TTY, optional RS-232C (TN-1710-16) or Parallel 8 Bit interfaces. A switch is provided for selecting the appropriate device. A column of three LED's above the device select rotary switch indicates which device has been selected. If the switch is placed in a position for which there is no device, the LED indicator for that device will not light.

The pushbutton on the module will illuminated when the TN-1710 is in the remote mode. Remote control is activated by pressing the pushbutton or by transmitting an X-ON character (also DCl, $\angle C T R L>Q$, ASCII code 21) through the device selected by the rotary switch. Once remote control has been activated any change in the device select knob will be ignored. When the remote control interface is ready to receive a command line it will transmit. a 非 (ASCII code 43). Each time a command line is received, a \# will be transmitted to indicate that it has completed the command and is ready to receive another command. Although the remote control interface will accept a new command between the reception of a command and the transmission of the \#, this is not a good practice and should only be used to abort Input or Output. Remote control is terminated by pressing the module pushbutton or by transmitting an X-OFF character (also called DC3, <CTRL> S, ASCII code 23).

The command string sent to the TN-1710-31 must be of the general form:

Command $[\mathrm{sp}]$ Argument $1 \quad[\mathrm{sp}] \ldots \ldots[\mathrm{sp}]$ Argument $\mathrm{N} \quad[\mathrm{cr}]$ or $[\mathrm{cr}]$

where $[\mathrm{sp}]$ is a space character (ASCII 40)

and $[\mathrm{Cr}]$ is a carriage return (ASCII 15)

Since the command string interpreter looks at only the first character and then ignores all following characters except a space or carriage return, the command may be a single letter, A, or many, ACQUIRE or AbC123\&. Al1 alphabetic characters must be capitalized. If the command string consists of just the command, it must be followed by a carriage return with no intervening spaces. If the command string has one or more arguments, the command and the first argument and all following arguments must be separated by at least one space. There can be no spaces between the last argument and the 
carriage return. Except where specified, all arguments must consist of numeric characters. Any non-numeric character in the argument field will result in an error.

Below is a listing of the command strings which can be used with the Remote Control Interface.

1. Acquire (See Appendix C)

A $[\mathrm{cr}]$

Stop acquisition

A [sp] Preset 1 [sp] ... [sp] Preset 6 [cr] Starts acquisition with preset parameters specified. The [cr] may be inserted after any of the arguments defaulting the remaining presets to whatever value they were last set to be.

2. BUG

$B[c r]$

Return the current location of the BUG and its contents. The location will be a seven digit number with leading zero suppression. The contents will be separated from the location by one space and will also be a seven digit number

B [sp] LLLL [cr]

Move the BUG to location $\mathrm{L}$ and return the new contents.

3. Clear

C $[\mathrm{cr}]$

Clear the last selected memory group.

4. Energy

E [sp] NNNN [cr]

Return the location of "energy" $\mathrm{N}$ as a seven digit number.

If the "energy" has digits to the right of the decimal

place, send all of them, including any trailing zeros. That is, send .005000 not .005 .

5. Group

G [cr]

Set the base group to what the front panel switch reads.

$G[\mathrm{sp}] \mathrm{S} / \mathrm{G}[\mathrm{cr}]$

Set the base group to the specified value.

6. Input

I $[\mathrm{cr}]$

Abort any ongoing input or output. 
$I$ [sp] D [cr]

Input spectral data from device D. D corresponds to the I/O device selector on the front panel of the TN-1710 such that:

$\begin{array}{llll}\text { TYPE }=0 & \text { MAG }=3 & \text { DISPLAY }=6 \\ \text { PEN }=1 & \text { PAPER }=4 & \cdots \\ \text { PRINT }=2 & \text { EIA }=5 & \end{array}$

7. Location

L [sp] NNNN [cr]

Return the "energy" of location $\mathrm{N}$ as a seven digit number including the decinal place.

8. Output

o [cr]

About any. ongoing input or output.

0 [sp] D [sp] M [cr]

Output spectral data to device $D$ in mode $M$. See the Input

command for device difinitions. The mode corresponds to the REGION/ FULL/INTEGRAL switch in the I/O control group.

ALL $=0 \quad$ REGION $=1 \quad$ INTEGRAL $=2$

9. Pointer

$P$ [cr]

Return the current contents of the pointer as a seven digit number and increment the pointer.

$\mathrm{P}$ [sp] LLLL [cr]

Move the pointer to location $L$, return the new contents, and increment the pointer. If remote control is disabled and re-enabled, the pointer may have been reset to 0 . Thus, it is advisable to reset the pointer if remote is re-enabled.

10. Region

$R$ [sp] X [ cr]

Select region number $\mathrm{X}$.

$R[s p] \quad X[s p] \quad N[r c]$

Select region number $X$ and return the net integral as an eight digit number.

$R$ [sp] X [sp] $G[c r]$

Select region number $X$ and return the gross integral.

$R$ [sp] X [sp] LOC1 [sp] LOC2 [cr]

Select region number $X$ and set its low limit to LOCl and its high limit to be LOC2. LOC2 must be greater than LOC1 and both limits should be in the selected base group. 
11. Status

$S$ [cr]

Return the current acquisition status.

If the system is not acquiring data, return a \#: If acquisition is in progress, return the number of live time seconds or number of sweeps elapsed as a seven digit number.

12. Transfer

$\mathrm{X}$ [sp] $\mathrm{FS} / \mathrm{FG}[\mathrm{sp}]+,-$, or $=[\mathrm{sp}] \mathrm{TS} / \mathrm{TG}[\mathrm{cr}]$

Set the "FROM" group to be FS/FG; set the transfer mode to $\operatorname{ADD}(+)$, DIRECT $(=)$, or SUB $(-)$; set the "TO" group to be TS/TG; and do the transfer.

13. Access CPU memory locations

a. [sp] NNNNNN= [cr.]

Return contents of CPU location $\mathrm{N}$ as a seven digit number.

a [sp] NNNNNN=X [cr]

Set CPU location $\mathrm{N}$ equal to $\mathrm{X}$.

This command is unprotected. No check is made to ensure CPU location $\mathrm{N}$ exists. Use only even values for $\mathrm{N}$. This command should only be used if you know exactly what you are doing and you are willing to suffer the consequences of turning power to the TN-1710 off and on again resulting in the loss of at least all data and region definitions.

14. Work Buffer

W [sp] NNNN [cr]

Return the start address of work buffer $\mathrm{N}$ as a seven digit

number.

$\mathrm{W} \cdot[\mathrm{sp}]$ NNNN [sp] LLL [cr]

Create or delete work buffer $N$. If $L$ is less than or equal

to zero delete the buffer. If $L$ is greater than zero create the buffer $L$ bytes long and return its start address.

Location is the offset of a spectral data memory address from the lowest address of the current group selected.

$A, O$, and $X$ may be prematurely terminated to default the remaining arguments to their last set value.

Upon receiving a $[c r]$ the remote control module will decode the command. If the command was not understood, the module will send a ?\#. If the command calls for the return of a value, the value will be computed and sent, followed by a \#. If no response is expected, as in the case of $\mathrm{X}$, a \# will be sent when the operation has been completed. 
To $\mathrm{TN}-1710-31$
G $1 / 1$ [cr]
G $2 / 4$ [cr]
G $2 / 3$ [cr]
G $3 / 16$ [cr]
A. $\varnothing 266[\mathrm{cr}]$

\section{B $[\mathrm{cr}]$}

$\mathrm{A} \varnothing 1 \varnothing \mathrm{A}[\mathrm{cr}]$

a $\varnothing \quad 99$ [cr]
Operation

set to full memory

set to 2nd quarter

nothing

nothing (if no 1710-28)

start acquisition in

the PHA mode for

100 secs.

send location of BUG

and its contents

nothing

nothing
From TN-1710-31

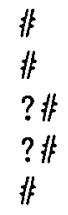

$100 \quad 234743$ \#

?\#

? 非

If the TN-1710 also has a. floppy disk (TN-1710-24), the terminal input cannot be used to drive the Remote Control Interface. In this case, if the rotary switch is placed in the TERMINAL position the LED will not light.

NOTE: The TN-1710-31 module may also interact with other modules which use the TYPE I/O port, such as the TN-1710-15 Isotope Markers module. There may be a conflict within the TN-1710 over the use of the teletype. These conflicts are easily resolved by selecting some other device, such as $\mathrm{RS}-232$, to be the remote control $\mathrm{I} / 0$ device. 
APPENDIX $C$

(To be used with TN-1710-31)

Definitions of Preset Parameters for Acquisition with a TN-1710-21

Preset 1

acquisition mode

Preset 2

Preset 3 preset scans or scans/trigger

preset integral
This value reflects the RESOLUTION, the array size, and the type of acquisition. The correct value to enter can be calculated by the following formula:

\section{$\mathrm{AM}+\mathrm{AS}+\mathrm{R}$}

where:

AM is the acquisition mode:

0 implies CONTINUOUS

8 implies TRIGGERED

AS is the array size code:

0 implies a 128 array

32 implies a 256 array

64 implies a 512 array

96 implies a 1024 array

$R$ is the resolution code:

0 implies a resolution of 64

256 implies a resolution of 128

512 implies a resolution of 256

768 implies a resolution of 512

1024 implies a resolution of 1024

This value should be the desired number of scans if the CONTINUOUS mode is specified or the number of scans/trigger if the TRIGGERED mode is specified. It must be a value between 1 and 4096 .

This is in the form of a mantissa +256* exponent where the mantissa is between 10 and 99 and the exponent is between 0 and 6 . A value of 0 implies infinity. 

$\begin{array}{ll}\text { Preset } 4 & \begin{array}{l}\text { preset exposure } \\ \text { time }\end{array}\end{array}$
The easiest way to calculate this value is to enable SETUP on the OPTICAL SPECTROMETER and select EXP TIME. Using the OMNI and the omni buttons select the desired exposure time and observe the values in the equation:

$E T=(M M M M * 10 \uparrow E+A A A A+2) * 10 \mu s$

MMM is the mantissa

$E$ is the exponent

AAAA is the array size

The value which must be sent for this preset is:

$(M M M / 2)-1+E * 4096$

Thus, if the desired exposure time is 1.00114 seconds the value sent should be:

$(996 / 2)-1+2 * 4096=8689$ for $a$ 512 array.

This value should always be 0 .

This value should be the desired number of triggers when the TRIGGERED mode is specified. It should be between 1 and 4096 . This value may be defaulted if the CONTINUOUS mode is specified. 
APPENDIX D

ה 


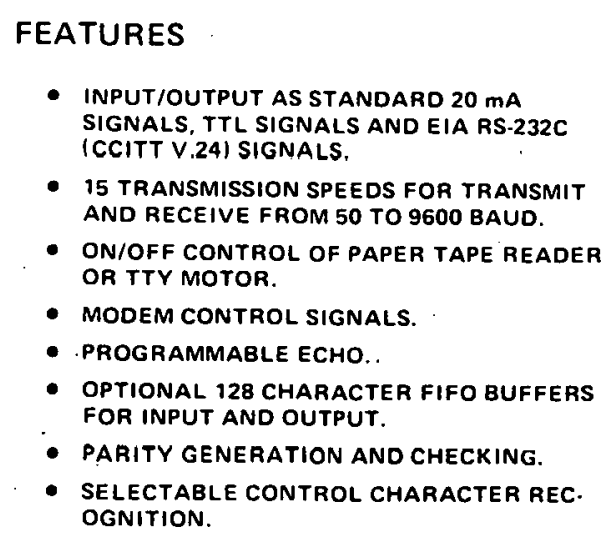

- INPUT/OUTPUT AS STANDARO 20 ma SIGNALS, TTL SIGNALS AND EIA RS-232C (CCITT V.24) SIGNALS.

- 15 TRANSMISSION SPEEDS FoR TRANSMIT AND RECEIVE FROM 50 TO 9600 BaUd.

- ON/OFF CONTROL OF PAPER TAPE READER OR TTY MOTOR.

- modem control signals.

- programmable echo..

- optional 128 Character fifo bUfFERS FOR INPUT AND OUTPUT.

- parity generation aNo checking. - Selectable control character rec.
ognition.

\author{
APPLICATIONS \\ - interface for a teletypewriter. \\ - interface for a crt terminal. \\ - interface for modem communication \\ LINK. \\ - data link between two camac systems. \\ - interface for character oriented \\ SERIAL EQUIPMENT.
}

\section{GENERAL DESCRIPTION}

The Mudel 3340 is a single-width CAMAC module that interfaces the CAMAC datuway directly to terminals. teletypewriters, modems, printers. etc. Characters are transmitted and received as $20 \mathrm{~mA}$ and TTL signals on one front panel connector and EIA RS-232C on the other front panel connector. Fifteen datid rutes are available: $50.75,110,134.5,150,300,600,1200,1800,2000,2400.3600,4800.7200 .9600$ Baud. Inpul and output data rates are independently adjustable via board mounted switches. The number of data bits in a character is switch selectable from 5 to 8 . Another switch option allows the choice of 1 or 2 stop bits. Parity error checking is also switch selectable.

A relay control signal is provided. This can be used to provide on/off control for a paper tape reader, a teletypewriter motor, or other load. The output is open collector and can drive loads up to 24 volts and 100 nilliamps. A fused +24 volt out put is provided for use with this circuit. Modem control flags are provided for establishing an automatic conmunication link through a modem via the EIA RS. $32 \mathrm{C}$ connector.

Optionally. two 128 character buffers can be provided. one for input and one for uutput. These buffers allow the data to be transferred a block at a time rather than character by character. Under progrant control the input can be echoed back to the output as well as to the computer.

\section{BLOCK TRANSFER MODE (FIFO option only)}

In order to operate in a block transfer mode, the End-Of-Block character must be selected un input and output ( 8 switches on output and 8 switches on input). The End-Of-Block character detection inust also be enabled.

On nutput hlnck transfers, the buffer is filled by doing write commands until $Q=0$ is detected. A $Q=0$ response means that the output buffer is full or the Fnd-Of-Block character was written. The modulc will then transmit the block of dat a at its selected Baud rate until the output buffer is empty.

Continued on following page 
BLOCK TRANSFER MODE (FIFO option only) - Cont'd.

On input block transfers, the input buffer is filled and a LAM is set, meaning that the input buffer is full or the End-Of-Block character has been written into the input buffer. The LAM is detected by the computer and it reads the input buffer until $Q=0$ is detected. A $Q=0$ response means that the input buffer is empty or the End-Of-Block character has been read.

When used with a teletypewriter, a carriage return character will generally be selected as the input End-Of-Block character, allowing the user to enter a line terminated by a carriage return. Likewise, a non-printing character, such as EOT, would be used to indicate the end of the output block (from the computer). When transferring blocks between CAMAC systems, a character such as EOT would be selected for both input and output. When selecting End-Of-Block characters, the parity bit of the sending device and receiving device code must match the switch selections.

\section{FUNCTION CODES}

\begin{tabular}{|l|c|c|c|}
\hline Command & 0 & $X$ & Action \\
\hline$F(0) \cdot A(0)$ & $A$ & 1 & Read the input register and clear LAM 2 and LAM 7. \\
\hline$F(1) \cdot A(0)$ & 1 & 1 & Read the status register. \\
\hline$F(1) \cdot A(12)$ & 1 & 1 & Read the LAM status register. \\
\hline$F(1) \cdot A(14)$ & 1 & 1 & Read the LAM request register. \\
\hline$F(8) \cdot A(15)$ & LAM & 1 & Test if a LAM is present. \\
\hline$F(9) \cdot A(0) \cdot S 1$ & 1 & 1 & Clear the buffer memories and UART. \\
\hline$F(16) \cdot A(0) \cdot S 1$ & $B$ & 1 & Write the output register and clear LAM 1. \\
\hline$F(17) \cdot A(0) \cdot S 1$ & 1 & 1 & Write the mode register. \\
\hline$F(17) \cdot A(13) \cdot S 1$ & 1 & 1 & Write the LAM mask register. \\
\hline$F(23) \cdot A(12) \cdot S 1$ & 1 & 1 & Selective clear the LAM status register. \\
\hline$Z \cdot S 2 \quad 0$ & 0 & $\begin{array}{l}\text { Clear LAM status register, LAM mask register, buffer memories. } \\
\text { mode register, and UART. }\end{array}$ \\
\hline Notes: A) $Q=0$ for buffer empty or selected input EOB character read. $Q=1$ otherwise. \\
B) $Q=0$ for bulfer full or selected output EOB character written. $Q=1$ otherwise. \\
\hline
\end{tabular}

\section{LAM REGISTER}

\begin{tabular}{|l|l|l|}
\hline Bit & Label & \\
\hline 11 & OR & Description \\
\hline 10 & Input buffer overrun (character has heen lost). \\
\hline 9 & PAR & Input character framing error detected. \\
\hline 8 & N/U & Not used. \\
\hline 7 & CP & Input character is availeble. \\
\hline 6 & CNP & Carrier Not Present (signal from data set). \\
\hline 5 & CTS & Clear To Send (signal from data set). \\
\hline 4 & DSR & Data Set Ready (signal from data set). \\
\hline 3 & RI & Ring Indicator (signal from data set). \\
\hline 2 & FULL & $\begin{array}{l}\text { Input buffer full or End-Of-Block character detected on input to the input buffer (buffer option } \\
\text { onlyl. }\end{array}$ \\
\hline 1 & EMP & Output buffer empty or output register emptr. \\
\hline Note: These bits represent edge triggered latches and thus indicate that the condition occured at least once since the last time that \\
\hline
\end{tabular}


MODE REGISTER (write only)

\begin{tabular}{|c|c|c|}
\hline Bit & Label & Description \\
\hline 7 & OUT EOB & Enables output End-Of.Block character detection. \\
\hline 6 & IN EOB & Enables input End-Of-Block character detection. \\
\hline 5 & TTY & Enables TTY control signal. \\
\hline 4 & DTR & Data Terminal Ready (signal to data set). \\
\hline 3 & RTS & Request To Send (signal to data set). \\
\hline 2 & IN DIS & Disables input data. \\
\hline 1 & ECHO & Enables echo of data input to Output. \\
\hline
\end{tabular}

STATUS REGISTER (read only)

\begin{tabular}{|c|c|c|}
\hline Bit & Label & Description \\
\hline 7 & TTY & TTY control signal enabled. \\
\hline 6 & CNP & Carrier Not Present (signal from data set). \\
\hline 5 & CTS & Clear to Send (signal from data set). \\
\hline 4 & DSR & Data Set Ready (signal from data set). \\
\hline 3 & RI & Ring Indicator (signal from data set). \\
\hline 2 & IN DIS & Input data disabled. \\
\hline 1 & ECHO & Echo of data input to output enabled. \\
\hline
\end{tabular}

\section{REGISTERS}

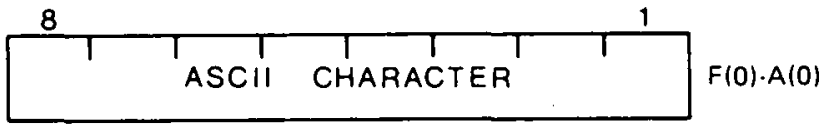

INPUT DATA

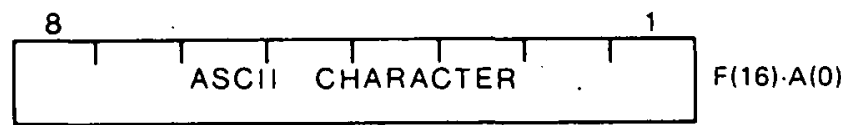

OUTPUT DATA

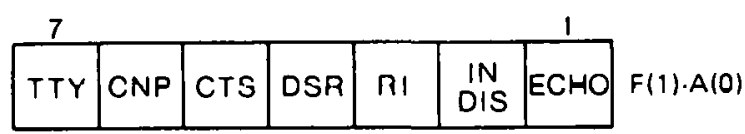

STATUS

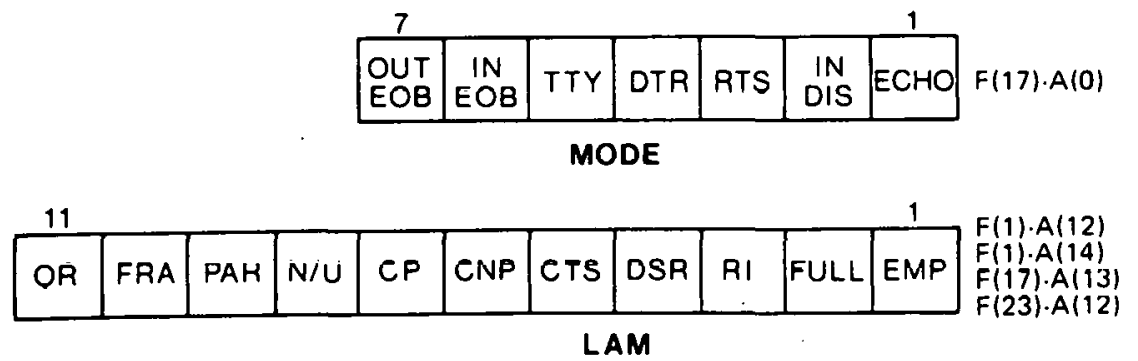




\section{INPUT/OUTPUT CONNECTORS}

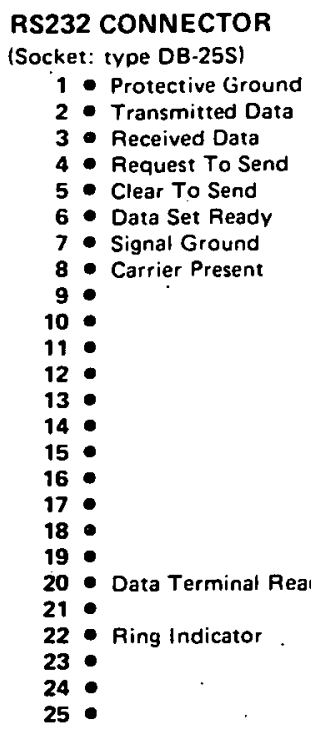

Mating Connector: DB.25P

Note: These signals are as specified by EIA RS232C or CCITT Recommendation V.24.

\section{TTY CONNECTOR}

(Socket: type DB-25S)

1 - Protective Ground

2 - 20 ma Receive Da

3 - 20 mA Tranșmit Data

4.

$5 \cdot$

$6 \cdot$

7. Signal Ground

- Relay Control Signal (Out)

$9 \cdot$

$10 \bullet$

11 - TTL Receive Data (In)

$12 \cdot$

13 - TTL Transmit Data

14 -

15. External X16 Transmit Clock

$16 \bullet$

17 - 20 mÁ Receive Data Return (In -)

$18 \cdot$

19.

20 - 20 mA Transmit Data Return (Out -)

$21 \cdot$ Fused $+24 \mathrm{~V}$

22 .

23 .

$24 \bullet$

$25 \bullet$

Mating Conniector: DB-25P

Note: TTL Receive Data and TTL Transmit Data are not factory strapped. To use these signals, install straps as indicated under STRAP SELECTABLE OPTIONS.
(Out)

$(\ln +1$

(Out +1

(In)

(Out)

\section{STRAP SELECTABLE OPTIONS}

$20 \mathrm{~mA}$ Driver Source or Sink Current

\begin{tabular}{ll} 
Source Current & Strap AA to BB \\
\hline & Strap CC to DD \\
\hline & 3tıap EE iu FF \\
\hline Sink Current & Strap AA to DD \\
\hline & Strap CC to FF \\
\hline
\end{tabular}

20 mA Receiver Source or Sink Current

Source Current

\begin{tabular}{ll} 
Source Current & Strap A to B \\
\hline & Strap C to D \\
\hline & Strap E to F \\
\hline Sink Current & Strap A to D \\
\hline & Strap C to F \\
\hline
\end{tabular}

Transmit Clock

Internal Clock

Strap 1

External Clock

TTL In

Strap TTL IN in order to use TTL input data.

\section{TL Out}

Strap TTL OUT in order to use.TTL output data. 
FRONT PANEL

LED Indicators
\begin{tabular}{ll}
$\frac{N}{L}$ & Flashes when the module is addressed. \\
\hline I/O Connectors & On when the L signal is true. \\
RS232 & D type connector 25.way cannon type DBC.25S or equivalent. \\
\hline$T T Y$ & D type connector $25 \cdot$ way cannon type DBC.25S or equivalent.
\end{tabular}

SWITCH SELECTABLE OPTIONS

TRANSMIT/RECEIVE BAUD RATES (S1 - S2) 4 BITS

\begin{tabular}{|c|c|c|c|c|}
\hline BAUD RATE & A & B & C & D \\
\hline 50 & 0 & 0 & 0 & 0 \\
\hline 75 & 1 & 0 & 0 & 0 \\
\hline 110 & 0 & 1 & 0 & 0 \\
\hline 134.5 & 1 & 1 & 0 & 0 \\
\hline 150 & 0 & 0 & 1 & 0 \\
\hline 300 & 1 & 0 & 1 & 0 \\
\hline 600 & 0 & 1 & 1 & 0 \\
\hline 1200 & 1 & 1 & 1 & 0 \\
\hline 1800 & 0 & 0 & 0 & 1 \\
\hline 2000 & 1 & 0 & 0 & 1 \\
\hline 2400 & 0 & 1 & 0 & 1 \\
\hline 3600 & 1 & 1 & 0 & 1 \\
\hline 4800 & 0 & 0 & 1 & 1 \\
\hline 7200 & 1 & 0 & 1 & 1 \\
\hline 9600 & 0 & 1 & 1 & 1 \\
\hline Note: Transmit and Receive Baud Rate Selection is com. \\
\hline pros
\end{tabular}
pletely separate.

$$
\text { "Qni" = } 0
$$

on switch.
OUTPUT END-OF-BLOCK CHARACTER (S3) 8 BITS

EXAMPLE:

\begin{tabular}{|l|l|l|l|l|l|l|l|l|}
\hline CHAR & 8 & 7 & 6 & 5 & 4 & 3 & 2 & 1 \\
\hline EOT & 0 & 0 & 0 & 0 & 0 & 1 & 0 & 0 \\
\hline
\end{tabular}

$E O T=A S C 11004$

INPUT END-OF-BLOCK CHARACTER (S4) 8 BITS

EXAMPLE:

\begin{tabular}{|l|l|l|l|l|l|l|l|l|}
\hline CHAR & 8 & 7 & 6 & 5 & 4 & 3 & 2 & 1 \\
\hline CR & 0 & 0 & 0 & 0 & 1 & 1 & 0 & 1 \\
\hline
\end{tabular}

$\mathrm{CR}=\mathrm{ASC} \| 015$

CONTROL SIGNALS (S5) 8 BITS

\begin{tabular}{|c|c|c|c|c|}
\hline Name & Symbol & \multicolumn{3}{|c|}{ Selection } \\
\hline Odd/Even Parity Select & EPS & \multicolumn{3}{|c|}{$\begin{array}{l}i=\text { Even parity } \\
0=\text { Odd parity }\end{array}$} \\
\hline Number of Bits/Character & $\begin{array}{l}\text { NB1 } \\
\text { NB2 }\end{array}$ & $\begin{array}{c}\text { NB2 } \\
0 \\
0 \\
1 \\
1\end{array}$ & $\begin{array}{c}\text { NB1 } \\
0 \\
1 \\
0 \\
.1\end{array}$ & $\begin{array}{c}\text { Bits/Character } \\
5 \\
6 \\
7 \\
8 \\
\end{array}$ \\
\hline Number of Stop Bits & TSB & \multicolumn{3}{|c|}{$\begin{array}{l}1=\text { Two stop bits/character } \\
0=\text { One stop bit/character }\end{array}$} \\
\hline No Parity & NP & \multicolumn{3}{|c|}{$\begin{array}{l}1=\text { Parity oliminated } T X \text { and } R X \\
0=\text { Normal parity } T X \text { and } R X\end{array}$} \\
\hline Input/Output Signal Standards & $\begin{array}{c}20 \mathrm{~mA} \\
\mathrm{RS} 232 \\
T \mathrm{TL}\end{array}$ & \multicolumn{3}{|c|}{$\begin{array}{l}\text { Set the switch for the signal standard being } \\
\text { used }=0 \text {, the other two switches }=1 \text {. }\end{array}$} \\
\hline
\end{tabular}


APPENDIX E 


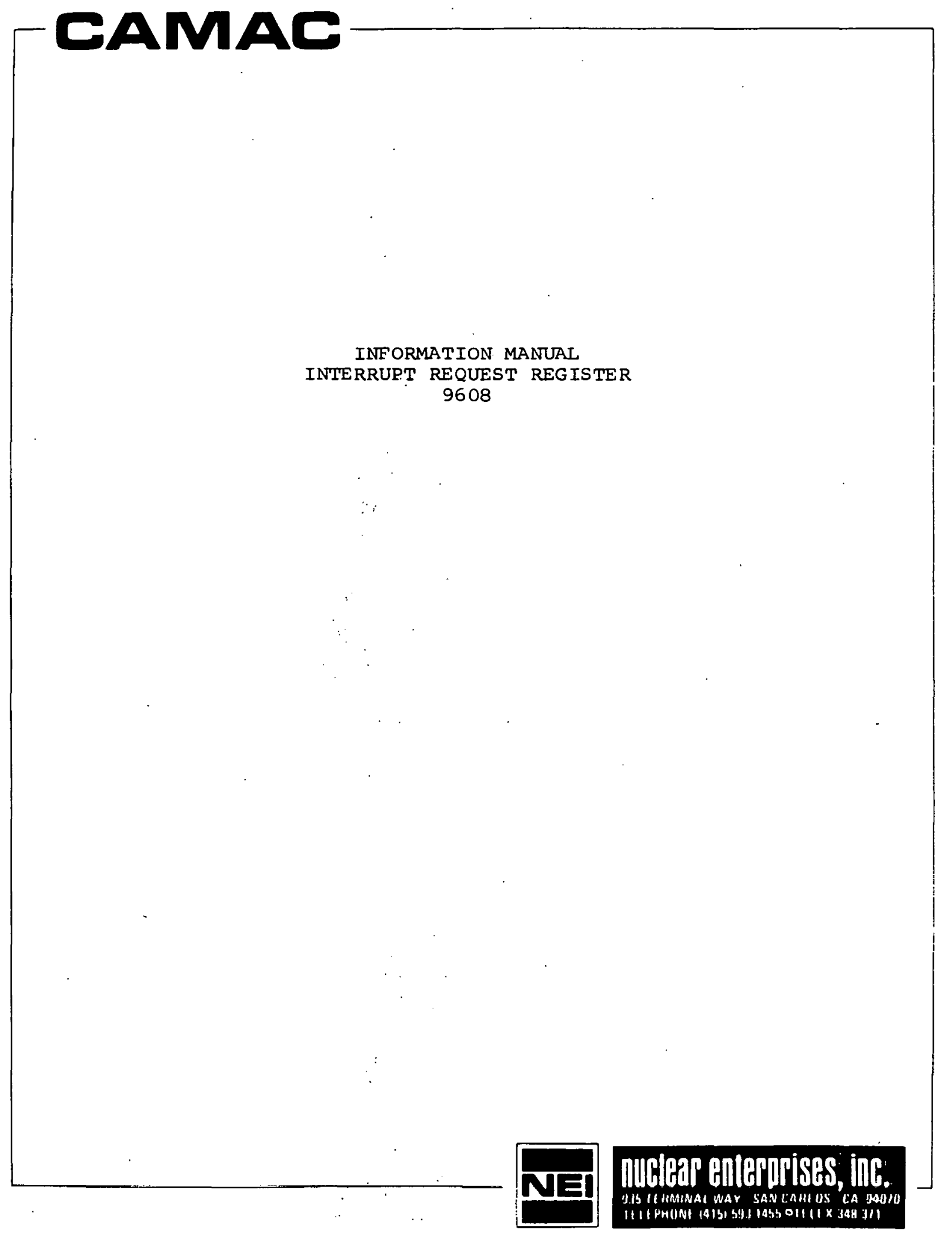


Notes on 9608

.P. Henline

April 18, 1980

1). Request lights will light only if mask light is on, but bits will be set

2) $\mathrm{A}(12) \mathrm{F}(11)$ affects only bits where mask light is on

3) $A(15) F(10)$ clears all request bits (whether they are masked or not)

4) $A(15) F(10)$ does not enable lam

5) on page 3, Status Register is same as Request Register

6) With lam disabled the mask register cannot be set and the request register can be set

7) Using CAMIK, after doing a test $\operatorname{lam}(\mathrm{A}(15) \mathrm{F}(8))$ or a test lam enable $(A(15) F(27))$,

ISTAT is even if on or enabled

ISTAT is odd if off or not enabled 
INFORMATION MANUAL

INTERRUPT REQUEST REGISTER

9608

USE OF DATAWAY:

Command Sub-Address and Function Codes

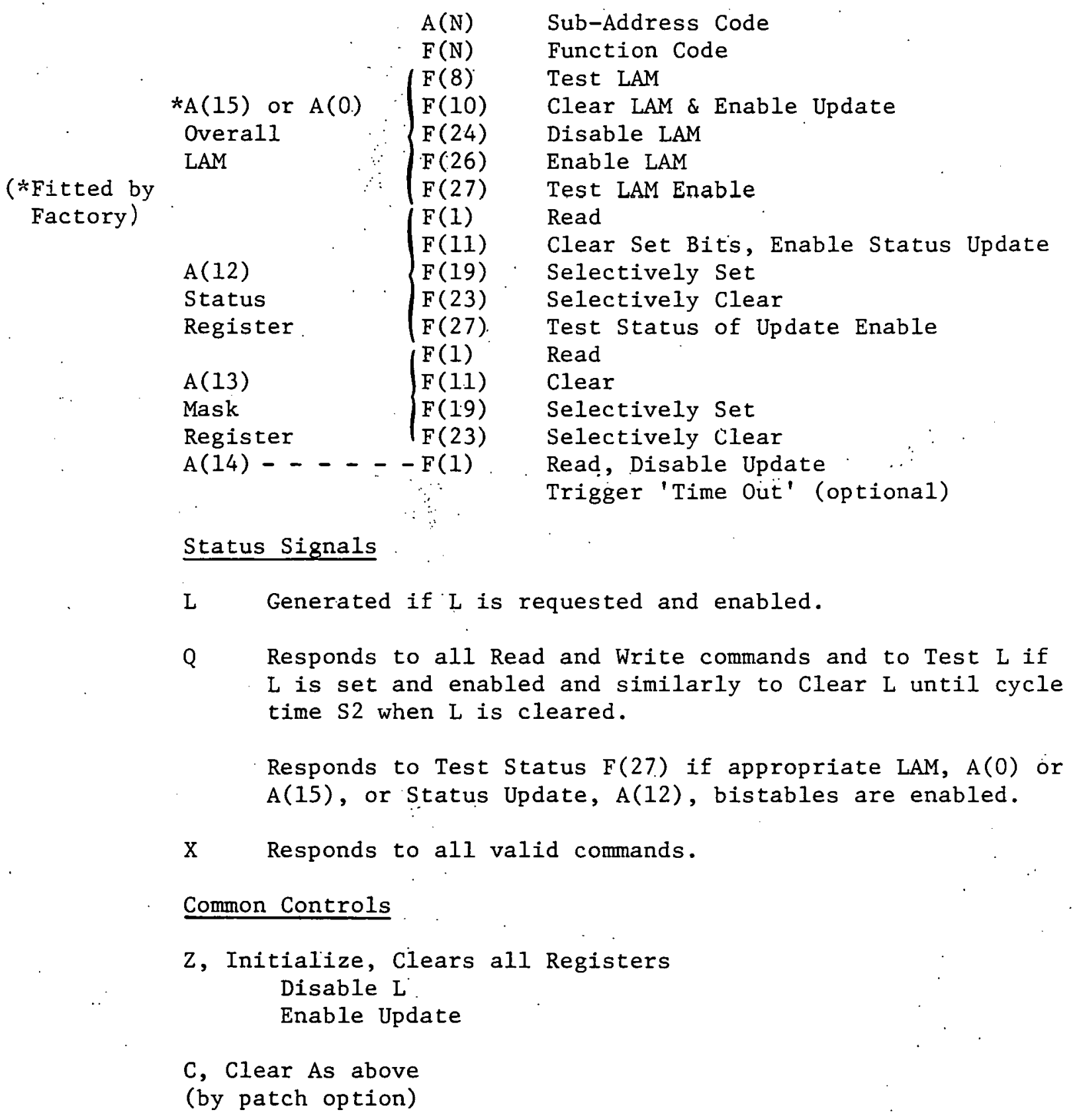




\section{OPERATING PROCEDURE}

Initializing

This command clears all the registers, disables LAM and enables transfer of data from the input Buffer to the status Register. When this command is terminated condition prevailing at the input will determine the state of the Buffer and transfer to the status Register is immediate.

\section{Status Register $A(12)$}

The total Status Register comprises an input Buffer Register and a Status Register. The Buffer Register is cleared on Initialise but will respond to input conditions when $Z$ terminates. The Buffer Register is d.c. coupled and the only data lost will be pulse inputs occurring concurrently with initialise command. The input Buffer will be reset when the data is transferred to the Status Register. (DC levels at the input will be re-entered.)

Updating of the Status Register is disabled whenever the module is being addressed and following a Read LAM Request command (see Request Register section). The status of the Update Enable can be ascertained by command Test Status $F(27)$. For diagnostic checkout the Status Register of each channel can be selectively set, F(19), or Selectively Cleared $\mathrm{F}(23)$.

Status Register is Read on command $F(1)$ and channels set which have been entered into the Request Register can be cleared by command $F(11)$ which will also enable the Update bistable.

\section{Mask Register $\dot{A}(13)$}

Interrupt inputs are subject to the Mask Register before entry into the Request Register and subsequent acknowledgement and clearance.

Channels of the Mask Register can be Selectively Set, F(19), and Selectively Cleared $F(23)$. The status of the Mask Register can be ascertained by the Read command $F(1)$ and is Cleared in total by command F(11).

The state of the Mask Register is displayed on the Front Panel.

\section{LAM Request A(14)}

Interrupt inputs which have been transferred to the Status Register and are enabled by the Mask Register will result in a LAM Request. LAM requests are Read by command $F(1)$ and the command disables update.

Update of the input request status can be achieved by one of the commands previously described either after a period defined by software 
INFORMATION MANUAL

INTERRUPT REQUEST REGISTER

9608

which will allow sufficient time to service the interrupt source or by abortion of the current state. If required a 'Time Out' facility described under USER OPTIONS can be used to re-enable the update bistable. The input Buffer Register ensures that any new interrupt signals are not lost and any interrupt levels remaining at the input due to an unsuccessful service routine will be re-entered.

The state of the Requests are displayed on the Front Panel.

Overall LAM $A(15)$ or $A(0)$

Any LAM Request will result in an Overall LAM signal which is displayed on the front panel. The Overall LAM signal is conditioned by a LAM enable/disable distable before generating a Dataway L signal.

Overall LAM can be tested by command TEST LAM F(8). The status of the LAM enable/disable bistable can be tested by $F(27)$. The conditioning bistable is Enabled by $F(26)$ and Disabled by $F(24)$. The Overall LAM can be cleared by $F(10)$ and this command also enables status update.

An Overall LAM signal is also available at the Front Panel $\sum$ L: This signal can be used to cause interrupts though systems have multilevel interrupt requirements but will be found useful in many applications.

\section{$\underline{\text { References }}$}

i CAMAC TID 25875 Section 5.4.1.

Fig. 11

ii CAMAC Supplementary Information TID 25877 Section K5.4.1.a

Fig. K5.4.1a-2

$$
\mathrm{K} 5 \cdot 4 \cdot 1 \mathrm{a}-3
$$

\section{CIRCUIT DESCRIPTION}

INTERRUPT CHANNELS

All eight interrupt channels are similar, this description pertains to Input 1.

Active inputs (clamp to ground) set the Input Buffer Register (U20, U30). This buffer is reset by Initialise command, $Z$, or when the input state is accepted by the Status Register (U20, U20). Interrupt is only accepted by the Status Register if the gate U30 is enabled by the Enable Status Update signal.

The Status Register can be selectively set or cleared by the appropriate command and the state of the Write through U10 line. (A '1' state on the Write Line is active $\bumpeq$ ov). The Status Register is Read by U50. 
Integrated curcuit $\mathrm{U} 40$ is the Mask Register which is cleared by the Clear Mask signal. The Mask can be selectively set or cleared through U10 and is read through $\mathrm{U} 30$. The state of the Mask Register is displayed by Ll (OV at pin $8 \mathrm{U} 40$ is the set state).

A Request signal is generated through $\mathrm{U} 30$ if an interrupt is received and the Mask is enabled. Requests are read through gate U50. If set LAM requests can be reset through $U 47$. The state of the Request is displayed by $\mathrm{L} 9$.

Write line WI is buffered by U6.

Waveforms pertain to active signals.

COMMAND DECODE

Conventional TTL decoding and encoding is used and the schematic diagram is sufficiently detailed for easy understanding. Waveforms pertain to active signals.

The 'Time Out' monostable U68 is triggered by the command Read Request. The delay time is determined by components R2 and C3 and obeys the approximate law

Delay $($ second $) \bumpeq 0.7 . \mathrm{C} 3(\mu \mathrm{F}) \cdot \mathrm{R} 2(1)$

When used to reenable the Update Bistable a suitable capacitor must be inserted in LK7. The value must be sufficiently high to ensure that the trailing edge of the delay signal from 'Time Out' rese'ts the Update Bistable but not high enough to prevent differentiation of the trailing edge. The value of the capacitor is related to C3. The factory fitted value of C3 is $1 \mathrm{uF}$ and for this value a suitable value to insert in $L K 7$ is $0.001 \mu \mathrm{F}$ ( IOV DC WKG). 

C. Armentrout
D. Drobnis
P. Henline
S. Izumi
S. Karin
J. Lohr
G. Shephard 